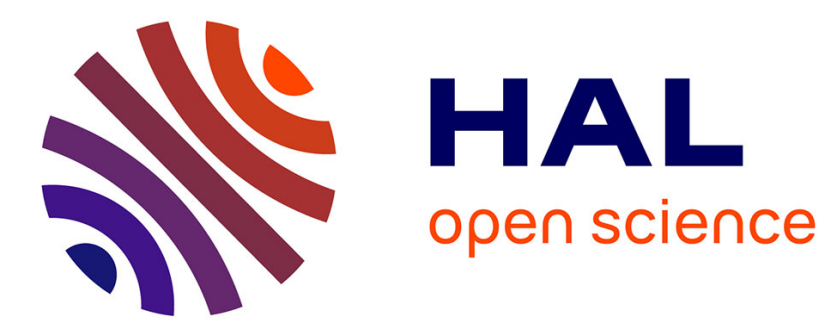

\title{
Collisionless magnetohydrodynamics with gyrokinetic effects
}

\author{
T. Passot, P.L. Sulem
}

\section{To cite this version:}

T. Passot, P.L. Sulem. Collisionless magnetohydrodynamics with gyrokinetic effects. Physics of Plasmas, 2007, 14, pp.2502. 10.1063/1.2751601 . hal-00388150

\section{HAL Id: hal-00388150 \\ https://hal.science/hal-00388150}

Submitted on 22 Jan 2022

HAL is a multi-disciplinary open access archive for the deposit and dissemination of scientific research documents, whether they are published or not. The documents may come from teaching and research institutions in France or abroad, or from public or private research centers.
L'archive ouverte pluridisciplinaire HAL, est destinée au dépôt et à la diffusion de documents scientifiques de niveau recherche, publiés ou non, émanant des établissements d'enseignement et de recherche français ou étrangers, des laboratoires publics ou privés. 


\title{
Collisionless magnetohydrodynamics with gyrokinetic effects
}

\author{
T. Passot and P. L. Sulem \\ Observatoire de la Côte d'Azur, CNRS, B.P. 4229, 06304 Nice Cedex 4, France
}

(Received 19 February 2007; accepted 31 May 2007; published online 16 August 2007)

\begin{abstract}
Anisotropic magnetohydrodynamics equations, which also capture the dynamics of quasi-transverse small scales obeying the gyrokinetic ordering, are derived using fourth-rank moment closures, based on a refined description of linear Landau damping and finite Larmor radius (FLR) corrections. This "FLR-Landau fluid model" reproduces the dispersion relation of low-frequency waves, up to scales that, in the case of quasi-transverse kinetic Alfvén waves, can be much smaller than the ion gyroradius. The mirror instability, which requires temperature anisotropy, is also captured, together with its quenching at small scales. This model that accurately reproduces the collisionless dissipation of low-frequency modes, should provide an efficient tool to simulate mesoscale turbulence in a magnetized collisionless plasma. (C) 2007 American Institute of Physics.
\end{abstract}

[DOI: $10.1063 / 1.2751601]$

\section{INTRODUCTION}

The solar wind and the terrestrial magnetosheath provide a natural laboratory for the study of magnetic turbulence in collisionless plasmas, owing to the high quality of in situ measurements performed on spacecraft exploration of the earth environment. Several basic questions arise from the analysis of these data. An example concerns the spectrum of the magnetic fluctuations, which displays a power-law behavior on a broad range of wavenumbers, with a conspicuous change of the spectral exponent near the inverse ion gyroradius. ${ }^{1-3}$ This property is often associated with the influence of wave dispersion, such as that due to the Hall term in the generalized Ohm's law. ${ }^{4-6}$ At these scales, however, kinetic effects cannot usually be neglected. It was also suggested that the steep magnetic spectral range, often referred to as a "dissipation range," could result from a superposition of cascades of kinetic Alfvén waves and of ion entropy fluctuations. ${ }^{7}$ Alfvenic turbulence is also believed to play an important role in the extended solar corona where kinetic dissipation can efficiently contribute to an anisotropic heating of the various particle species. ${ }^{8}$

In addition to turbulence properties, an open question concerns the formation and the role of various coherent structures involving typical scales of a few ion gyroradii $r_{L}$, observed in the magnetosheath and identified as mirror modes, magnetic solitons, shocklets, or Alfvenic filaments. These latter structures, most often observed behind quasiperpendicular shocks, have in particular been associated with the "bump" visible on the magnetic energy spectrum at scales slightly larger than the ion Larmor radius.

Another important question concerns collisionless reconnection that takes place in the magnetopause and the magnetotail, ${ }^{12,13}$ where wave-particle resonances and finite Larmor radius (FLR) effects could have a non-negligible influence on the breaking of the ideal character of the plasma. $^{14,15}$

A fully kinetic approach could appear necessary to address the above issues. In the context of space and astrophysical turbulence, important efforts are indeed presently made $^{7,9}$ to use the gyrokinetic theory previously developed for fusion plasmas. By averaging over the gyromotion of the particles, this approach filters out the shortest time scales and isolates low-frequency phenomena. It also assumes a strong scale anisotropy resulting from the quasi-transverse dynamics that should develop at small scales in a strongly magnetized magnetohydrodynamic (MHD) turbulence. ${ }^{10}$ Nevertheless, it is not entirely clear that the turbulent cascade does not also involve energy transfer to small parallel scales and that the role of longitudinally propagating modes of whistler type can be totally ruled out. ${ }^{11}$ Discriminating between the different processes at the origin of the observed magnetic spectra remains, in fact, a challenging question.

The gyrokinetic theory appears as a powerful tool that has the great advantage of being asymptotically exact. Nevertheless, turbulent regimes that involve a broad spectral range are hardly amenable to kinetic simulations, even within the gyrokinetic asymptotics. Furthermore, this approach concentrates on scales comparable to, or smaller than, the ion gyroradius and does not capture the dynamics of larger MHD scales.

In order to study the range of scales associated with the transition between the magnetohydrodynamic and the kinetic scales, one is led to look for a fluid description that extends the usual MHD by retaining relevant kinetic effects. This approach cannot be fully systematic but, in many instances where kinetic effects are present although not dominant over hydrodynamic processes, it may provide a reasonable description of the dynamics. This approach was initiated in Ref. 16 for MHD scales asymptotically large compared with the ion gyroradius, by closing the fluid hierarchy derived from the drift kinetic equation in a way consistent with the linear kinetic theory. More recently, leading-order corrections resulting from finite Larmor radius (FLR) effects were evaluated perturbatively within a fluid description (see Ref. 17 and references therein), in order to reproduce the dispersive character of MHD waves whose typical length scale is not asymptotically large relative to $r_{\mathrm{L}}$. Nevertheless, in many instances, much smaller transverse scales have to be retained. 
For this purpose, we discuss in this paper a new Landau fluid model that retains transverse scales comparable to, or even significantly smaller than, the ion gyroradius. In contrast with the gyrofluids obtained by taking the moments of the gyrokinetic equation, this "FLR-Landau fluid" model preserves the global structure of the usual MHD equations with anisotropic pressures, and it does not assume a quasitransverse dynamics at all the scales. Nevertheless, the model only retains a linear (or quasi-linear) description of FLR effects. The nongyrotropic contributions are indeed expressed in terms of low-order fluid moments and of the magnetic field fluctuations, in a way consistent with the low-frequency linear kinetic theory, both for scales obeying the gyrokinetic ordering $\left(\omega / \Omega \sim k_{\|} / k_{\perp} \ll 1\right.$, with $\left.k_{\perp} r_{\mathrm{L}} \sim 1\right)$ and for isotropic large scales $\left(k_{\perp} r_{\mathrm{L}} \sim k_{\|} r_{\mathrm{L}} \ll 1\right)$, for which the usual FLR corrections $^{17}$ are recovered as the leading order in a longwavelength expansion. Similar to gyrofluids, our model faces the closure problem of the hierarchy of the moment equations, that both approaches address by neglecting particle trapping and treating Landau damping at a linear level only. Nevertheless, in spite of the linear description of the kinetic effects, the accurate damping of the small-scale modes provides a realistic sink for the energy cascade. Numerical integration of the FLR-Landau fluid does not in particular require artificial dissipation that could also affect the smallest scales of the cascade and the transition to the dissipation range, possibly enhancing the bottleneck effect, as observed in the different but nevertheless related context of strongly compressible turbulence. ${ }^{18}$

The paper is organized as follows. Section II briefly reviews the equations for the fluid moments, as derived from Vlasov-Maxwell equations. The closure problems associated with the determination of the fourth-rank cumulants and of the nongyrotropic contributions of the various fields are addressed in Secs. III and IV, on the basis of the linear kinetic theory for low-frequency perturbations. Section V deals with the validation of the model whose predictions are compared with the results of the full linear kinetic theory, for the dispersion and the noncollisional damping of Alfvén and magnetosonic waves propagating in various directions. It also addresses the mirror instability that can develop in a plasma where the ion perpendicular temperature is higher than the parallel one. Section VI provides a brief conclusion.

\section{THE FLUID HIERARCHY}

Consider a spatially homogeneous uniformly magnetized plasma with a bi-Maxwellian equilibrium distribution function for particles of species $r$ with charge $q_{r}$, mass $m_{r}$, and the same averaged number density $n^{(0)}$, in the absence of net charges or currents.

In a regime where the Alfvén wave velocity is small relative to the light speed, the plasma is electrically neutral and the displacement current can also be neglected (Appendix A). One then defines the proton density $\rho_{p}=m_{p} n$, and neglects terms proportional to the mass ratio $m_{e} / m_{p}$. The proton and electron velocities are related by $\vec{u}_{e}=\vec{u}_{p}-\vec{j} /(e n)$, where the current is given by $\vec{j}=(c / 4 \pi) \nabla \times \vec{B}$. One has the usual equations

$$
\begin{aligned}
& \partial_{t} \rho_{p}+\nabla \cdot\left(\rho_{p} \vec{u}_{p}\right)=0, \\
& \partial_{t} \vec{u}_{p}+\vec{u}_{p} \cdot \nabla \vec{u}_{p}+\frac{1}{\rho_{p}} \nabla \cdot \mathbf{p}_{p}-\frac{e}{m_{p}}\left(\vec{E}+\frac{1}{c} \vec{u}_{p} \times \vec{B}\right)=0, \\
& \vec{E}=-\frac{1}{c}\left(\vec{u}_{p}-\frac{\vec{j}}{n e}\right) \times \vec{B}-\frac{1}{n e} \nabla \cdot \mathbf{p}_{e},
\end{aligned}
$$

together with the Faraday-Maxwell equation $\partial_{t} \vec{B}=-c \nabla \times \vec{E}$. Here and in the following, tensors of rank 2 or more are bolded.

The ion pressure tensor is rewritten as the sum of gyrotropic and gyroviscous contributions $\mathbf{p}_{p}=p_{\perp p} \mathbf{n}+p_{\| p} \boldsymbol{\tau}+\boldsymbol{\Pi}$, with $\mathbf{n}=\mathbf{I}-\hat{b} \otimes \hat{b}$ and $\boldsymbol{\tau}=\hat{b} \otimes \hat{b}$, where $\hat{b}=\vec{B} /|\vec{B}|$ is the unit vector along the local magnetic field. The electron pressure tensor is taken gyrotropic and characterized by the parallel and transverse pressures $p_{\| e}$ and $p_{\perp e}$.

To simplify the writing, we drop the species index and write the equations governing the perpendicular and parallel pressures of the ions in the form

$$
\begin{aligned}
\partial_{t} p_{\perp} & +\nabla \cdot\left(\vec{u} p_{\perp}\right)+p_{\perp} \nabla \cdot \vec{u}-p_{\perp} \hat{b} \cdot \nabla \vec{u} \cdot \hat{b} \\
& +\frac{1}{2}(\operatorname{tr} \nabla \cdot \mathbf{q}-\hat{b} \cdot(\nabla \cdot \mathbf{q}) \cdot \hat{b}) \\
& +\frac{1}{2}\left(\operatorname{tr}(\boldsymbol{\Pi} \cdot \nabla \vec{u})^{S}-(\boldsymbol{\Pi} \cdot \nabla \vec{u})^{S}: \boldsymbol{\tau}+\boldsymbol{\Pi}: \frac{d \boldsymbol{\tau}}{d t}\right)=0, \\
\partial_{t} p_{\|}+ & \nabla \cdot\left(\vec{u} p_{\|}\right)+2 p_{\|} \hat{b} \cdot \nabla \vec{u} \cdot \hat{b}+\hat{b} \cdot(\nabla \cdot \mathbf{q}) \cdot \hat{b} \\
& +(\boldsymbol{\Pi} \cdot \nabla \vec{u})^{S}: \boldsymbol{\tau}-\boldsymbol{\Pi}: \frac{d \boldsymbol{\tau}}{d t}=0,
\end{aligned}
$$

where $\mathbf{q}$ is the heat flux tensor. A main point of the present model is that corrections to the usual anisotropic MHD equations are retained at a linear level only, thus suggesting to neglect the contributions of the gyroviscous stress tensor arising in Eqs. (4) and (5). It is nevertheless of interest to note that retaining the coupling to the gyroviscous stress is needed to ensure exact energy conservation, whatever the form of the forthcoming closure of the fluid hierarchy. ${ }^{19}$

It is convenient to decompose the proton heat flux tensor by writing $\mathbf{q}=\mathbf{S}+\boldsymbol{\sigma}$ with the conditions $\sigma_{i j k} n_{j k}=0$ and $\sigma_{i j k} \tau_{j k}=0$. One has

$$
\begin{aligned}
S_{i j k}= & \frac{1}{2}\left(S_{i}^{\perp} n_{j k}+S_{j}^{\perp} n_{i k}+S_{k}^{\perp} n_{i j}+S_{l}^{\perp} \tau_{l i} n_{j k}+S_{l}^{\perp} \tau_{l j} n_{i k}\right. \\
& \left.+S_{l}^{\perp} \tau_{l k} n_{i j}\right)+S_{i}^{\|} \tau_{j k}+S_{j}^{\|} \tau_{i k}+S_{k}^{\|} \tau_{i j}-\frac{2}{3}\left(S_{l}^{\|} \tau_{l i} \tau_{j k}\right. \\
& \left.+S_{l}^{\|} \tau_{l j} \tau_{i k}+S_{l}^{\|} \tau_{l k} \tau_{i j}\right),
\end{aligned}
$$

where $S_{i}^{\|}=q_{i j k} \tau_{j k}$ and $S_{i}^{\perp}=q_{i j k} n_{j k} / 2$ are the (vector) components of the fluxes of parallel and transverse heat $\vec{S}^{\|}$and $\vec{S}^{\perp}$, respectively. The parallel fluxes of perpendicular and parallel heat that are the only contributions to the gyrotropic heat flux tensor, are given by $q_{\perp}=\vec{S}^{\perp} \cdot \hat{b}$ and $q_{\|}=\vec{S}^{\|} \cdot \hat{b}$, respectively. We thus write $\vec{S}^{\perp}=q_{\perp} \hat{b}+\vec{S}_{\perp}^{\perp}$ and $\vec{S}^{\|}=q_{\|} \hat{b}+\vec{S}_{\perp}^{\|}$, where the perpendicular fluxes of perpendicular and parallel heat $\vec{S}_{\perp}^{\perp}$ and $\vec{S}_{\perp}^{\|}$, respectively, are computed in a linearized approximation and 
are thus viewed as perpendicular to the ambient field $B_{0} \hat{z}$.

One easily checks that since at the level of the linear approximation, $\hat{b} \cdot(\nabla \cdot \boldsymbol{\sigma}) \cdot \hat{b} \approx \partial_{i}\left(\sigma_{i j k} \tau_{i j}\right)=0$ and $[\operatorname{tr}(\nabla \cdot \boldsymbol{\sigma})$ $-\hat{b} \cdot(\nabla \cdot \boldsymbol{\sigma}) \cdot \hat{b}] \approx \partial_{i}\left(\sigma_{i j k} n_{i j}\right)=0$, it is sufficient to only retain the $S_{i j k}$ contribution to $q_{i j k}$, and write

$$
\begin{aligned}
& \hat{b} \cdot(\nabla \cdot \mathbf{q}) \cdot \hat{b} \approx \nabla \cdot\left(q_{\|} \hat{b}+\vec{S}_{\perp}^{\|}\right)-2 q_{\perp} \nabla \cdot \hat{b} \\
& \frac{1}{2}[\operatorname{tr}(\nabla \cdot \mathbf{q})-\hat{b} \cdot(\nabla \cdot \mathbf{q}) \cdot \hat{b}] \approx \nabla \cdot\left(q_{\perp} \hat{b}+\vec{S}_{\perp}^{\perp}\right)+q_{\perp} \nabla \cdot \hat{b} .
\end{aligned}
$$

The equation governing the evolution of the heat flux tensor involves the fourth-rank moment $\mathbf{r}$. Instead of dealing with this quantity, it may be convenient to isolate the cumulant $\widetilde{\mathbf{r}}$ by writing

$$
\begin{aligned}
\rho r_{i j k l}= & P_{i j} P_{l k}+P_{i k} P_{j l}+P_{i l} P_{j k}+P_{i j} \Pi_{l k}+P_{i k} \Pi_{j l}+P_{i l} \Pi_{j k} \\
& +\Pi_{i j} P_{l k}+\Pi_{i k} P_{j l}+\Pi_{i l} P_{j k}+\rho \tilde{r}_{i j k l} .
\end{aligned}
$$

Since FLR corrections are retained at the linear level only, the contribution $\Pi_{i j} \Pi_{l k}+\Pi_{i k} \Pi_{j l}+\Pi_{i l} \Pi_{j k}$ has been neglected in Eq. (9). The gyrotropic part of $\widetilde{\mathbf{r}}$ is characterized by three parameters: $\widetilde{r}_{||||}=\widetilde{r}_{i j l k} \tau_{i j} \tau_{k l}, \quad \tilde{r}_{\perp \|}=\frac{1}{2} \widetilde{r}_{i j l k} n_{i j} \tau_{k l}$, and $\widetilde{r}_{\perp \perp}=\frac{1}{4} \widetilde{r}_{i j l k} n_{i j} n_{k l}$, which are related to $r_{\|||}=r_{i j l k} \tau_{i j} \tau_{k l}$, $r_{\perp \|}=\frac{1}{2} r_{i j l k} n_{i j} \tau_{k l}$, and $r_{\perp \perp}=\frac{1}{4} r_{i j l k} n_{i j} n_{k l}$ by

$$
\begin{aligned}
& \tilde{r}_{|| \mid}=r_{|| \mid}-3 \frac{p_{\|}^{2}}{\rho}, \\
& \tilde{r}_{\| \perp}=r_{\| \perp}-\frac{p_{\perp} p_{\|}}{\rho}, \\
& \tilde{r}_{\perp \perp}=r_{\perp \perp}-2 \frac{p_{\perp}^{2}}{\rho} .
\end{aligned}
$$

One has

$$
\begin{aligned}
\tilde{r}_{i j k l}= & \frac{\tilde{r}_{\| l l}}{3}\left(\tau_{i j} \tau_{k l}+\tau_{i k} \tau_{j l}+\tau_{i l} \tau_{j k}\right) \\
& +\widetilde{r}_{\| \perp}\left(n_{i j} \tau_{k l}+n_{i k} \tau_{j l}+n_{i l} \tau_{j k}+\tau_{i j} n_{k l}+\tau_{i k} n_{j l}+\tau_{i l} n_{j k}\right) \\
& +\frac{\widetilde{r}_{\perp \perp}}{2}\left(n_{i j} n_{k l}+n_{i k} n_{j l}+n_{i l} n_{j k}\right)+\widetilde{r}_{i j k l}^{\mathrm{NG}}
\end{aligned}
$$

where $\widetilde{r}_{i j k l}^{\mathrm{NG}}$ refers to the nongyrotropic contribution of $\widetilde{r}_{i j k l}$.

The equations for the gyrotropic heat fluxes read (see Refs. 17 and 19)

$$
\begin{aligned}
\partial_{t} q_{\|} & +\nabla \cdot\left(q_{\|} \vec{u}\right)+3 q_{\|} \hat{b} \cdot \nabla \vec{u} \cdot \hat{b}+3 p_{\|}(\hat{b} \cdot \nabla)\left(\frac{p_{\|}}{\rho}\right) \\
& +\nabla \cdot\left(\widetilde{r}_{\|\mid\|} \hat{b}\right)-3 \widetilde{r}_{\| \perp} \nabla \cdot \hat{b}+\partial_{z} R_{\|}^{\mathrm{NG}}=0, \\
\partial_{t} q_{\perp} & +\nabla \cdot\left(\vec{u} q_{\perp}\right)+q_{\perp} \nabla \cdot \vec{u}+p_{\|}(\hat{b} \cdot \nabla)\left(\frac{p_{\perp}}{\rho}\right)+\frac{p_{\perp}}{\rho}\left(\partial_{x} \Pi_{x z}\right. \\
& \left.+\partial_{y} \Pi_{y z}\right)+\nabla \cdot\left(\widetilde{r}_{\| \perp} \hat{b}\right)+\left(\left(p_{\|}-p_{\perp}\right) \frac{p_{\perp}}{\rho}-\widetilde{r}_{\perp \perp}+\widetilde{r}_{\| \perp}\right) \\
& \times(\nabla \cdot \hat{b})+\partial_{z} R_{\perp}^{\mathrm{NG}}=0,
\end{aligned}
$$

where $R_{\|}^{\mathrm{NG}}$ and $R_{\perp}^{\mathrm{NG}}$ stand for the contributions originating from the nongyrotropic parts of the fourth-order moments.

To close the fluid hierarchy, it is necessary to express both the gyrotropic fourth-rank cumulants and the nongyrotropic contributions to the various moments in terms of known quantities.

\section{FOURTH-RANK CUMULANT CLOSURE}

The closure procedure consists in expressing the fourthrank cumulants in terms of lower order moments and possibly of the magnetic fluctuations, within the framework of the low-frequency linear kinetic theory. It also involves the replacement of the plasma response function arising in the resulting expressions, by a suitable Padé approximant whose number of poles is a compromise between accuracy and excessive complexity. For a prescribed pole number, the approximant is however not unique, depending on the allotment of the fitting conditions at zero and infinity and resulting in a different accuracy for the global fit of the function. 20

\section{A. Modeling of $\tilde{\boldsymbol{r}}_{||||}$}

To model $\widetilde{r}_{\|\mid\|}$, one proceeds as in Ref. 16 (see also Ref. 17). Comparing the expression of $\widetilde{r}_{\|\|}^{(1)}$, with those of $S_{z}^{\|}$or $T_{\|}^{(1)}$ provided by the kinetic theory (see Appendix B), one gets

$$
\begin{aligned}
\tilde{r}_{\||| \mid} & =\sqrt{\frac{2 T_{\|}^{(0)}}{m}} \frac{2 \zeta^{2}\left[1+2 \zeta^{2} R(\zeta)\right]+3[R(\zeta)-1]-12 \zeta^{2} R(\zeta)}{2 \zeta \operatorname{sgn}\left(k_{z}\right)\left[1-3 R(\zeta)+2 \zeta^{2} R(\zeta)\right]} S_{z}^{\|} \\
& \equiv \sqrt{\frac{2 T_{\|}^{(0)}}{m}} \mathcal{F}_{S} S_{z}^{\|}
\end{aligned}
$$

and

$$
\begin{aligned}
\tilde{r}_{\|\|} & =\frac{p_{\|}^{(0)} T_{\|}^{(0)}}{m} \frac{2 \zeta^{2}\left[1+2 \zeta^{2} R(\zeta)\right]+3[R(\zeta)-1]-12 \zeta^{2} R(\zeta)}{1-R(\zeta)+2 \zeta^{2} R(\zeta)} \frac{T_{\|}^{(1)}}{T_{\|}^{(0)}} \\
& \equiv \frac{p_{\|}^{(0)} T_{\|}^{(0)}}{m} \mathcal{F}_{T} \frac{T_{\|}^{(1)}}{T_{\|}^{(0)}} .
\end{aligned}
$$

Here, $R$ is the plasma response function related to the usual plasma dispersion function $Z(\zeta)$ by $R(\zeta)=1+\zeta Z(\zeta)$, with $\zeta=\left(\omega /\left|k_{z}\right|\right) \sqrt{m / 2 T_{\|}^{(0)}}$. The superscripts $(0)$ and (1) refer to the equilibrium state and the perturbation, respectively.

One then notices that when replacing the plasma response function $R$ by the four-pole Padé approximant

$R_{4}(\zeta)=\frac{4-2 i \sqrt{\pi} \zeta+(8-3 \pi) \zeta^{2}}{4-6 i \sqrt{\pi} \zeta+(16-9 \pi) \zeta^{2}+4 i \sqrt{\pi} \zeta^{3}+(6 \pi-16) \zeta^{4}}$,

one has the identity

$$
\lambda \frac{\mathcal{F}_{S}}{\mathcal{F}_{T}}+i \mu \frac{k_{z}}{\left|k_{z}\right|}=\mathcal{F}_{S}
$$

with $\lambda=(32-9 \pi) /(3 \pi-8)$ and $\mu=-2 \sqrt{\pi} /(3 \pi-8)$. This leads to the closure relation 


$$
\tilde{r}_{\| \mid}=\lambda p_{\|}^{(0)} \frac{T_{\|}^{(0)}}{m} \frac{T_{\|}^{(1)}}{T_{\|}^{(0)}}+\mu \sqrt{\frac{2 T_{\|}^{(0)}}{m}} \frac{i k_{z}}{\left|k_{z}\right|} S_{z}^{\|} .
$$

It is noticeable that the above choice for the four-pole Padé approximant seems to be the only one leading to a closure relation of the form (19).

\section{B. Modeling of $\tilde{r}_{\| \perp}$}

The cumulant $\widetilde{r}_{\| \perp}$ can be expressed in terms of $q_{\perp}$ and the parallel current $j_{z}$. One has

$$
\begin{aligned}
\tilde{r}_{\| \perp}= & \sqrt{\frac{2 T_{\|}^{(0)}}{m}} \frac{1-R(\zeta)+2 \zeta^{2} R(\zeta)}{2 \zeta R(\zeta)}\left[q_{\perp}+\left[\Gamma_{0}(b)-\Gamma_{1}(b)\right]\right. \\
& \left.\times \frac{p_{\perp}^{(0)} p_{\|}^{(0)}}{\rho^{(0)} v_{\mathrm{A}}^{2}}\left(\frac{T_{\perp}^{(0)}}{T_{\|}^{(0)}}-1\right) \frac{j_{z}}{e n^{(0)}}\right]
\end{aligned}
$$

where $v_{\mathrm{A}}=B_{0} / \sqrt{4 \pi \rho^{(0)}}$ is the Alfvén velocity and $\rho^{(0)}$ the plasma density at equilibrium. Furthermore, $\Gamma_{\nu}(b)=e^{-b} I_{\nu}(b)$, where $I_{\nu}$ is the modified Bessel function of order $\nu$, and $b=T_{\perp}^{(0)} k_{\perp}^{2} / m \Omega^{2}=k_{\perp}^{2} r_{\mathrm{L}}^{2} / 2$. Here, $r_{\mathrm{L}}$ denotes the particle Larmor radius.

It is convenient at this step to use a three-pole Padé approximant of the function $R$. As discussed below, several choices are possible for the coefficients $a_{0}$ (which is purely imaginary and thus conveniently written $\left.\left|a_{0}\right| i\right), a_{1}$, and $a_{2}$ arising in the three-pole Padé approximant

$$
R_{3}(\zeta)=\frac{-\frac{1}{2} \zeta-a_{0}}{\zeta^{3}-a_{2} \zeta^{2}-a_{1} \zeta-a_{0}} .
$$

Whatever this choice, one has

$$
\frac{1-R_{3}(\zeta)+2 \zeta^{2} R_{3}(\zeta)}{2 \zeta R_{3}(\zeta)}=\frac{a_{1}-1 / 2}{\zeta+2 a_{0}}=\frac{a_{1}-1 / 2}{\frac{\omega}{k_{z}} \sqrt{\frac{m}{2 T_{\|}^{(0)}}}+2\left|a_{0}\right| i} .
$$

Noting that when coming back to physical variables (both in time and space), $\omega$ transforms into $i \partial_{t}, k_{z}$ into $-i \partial_{z}$ and $i \operatorname{sgn} k_{z}$ into the Hilbert transform $\mathcal{H}_{z}$ relative to the $z$ coordinate, it follows that substituting (23) into Eq. (21) leads to the evolution equation

$$
\begin{aligned}
\left(\frac{d}{d t}\right. & \left.-2\left|a_{0}\right| \sqrt{\frac{2 T_{\|}^{(0)}}{m}} \mathcal{H}_{z} \partial_{z}\right) \widetilde{r}_{\| \perp}+\frac{2 T_{\|}^{(0)}}{m}\left(a_{1}-\frac{1}{2}\right) \\
& \times \partial_{z}\left[q_{\perp}+\left[\Gamma_{0}(b)-\Gamma_{1}(b)\right] \frac{p_{\perp}^{(0)}}{v_{A}^{2}}\left(\frac{T_{\perp}^{(0)}-T_{\|}^{(0)}}{m_{p}}\right) \frac{j_{z}}{e n^{(0)}}\right]=0 .
\end{aligned}
$$

The notation $m_{p}$ is used in the term where the proton mass is to remain unchanged when turning to the corresponding equation for the electron (where $e$ is to be changed into $-e$ ). The convective derivative has been reintroduced to ensure Galilean invariance.

The values of the coefficients entering the Padé approximant of $R_{3}$ used in Refs. 16 and 17 and referred to as $R_{3,2}$ in Ref. 20 are $a_{0}=i / \sqrt{\pi}, a_{1}=3 / 2$, and $a_{2}=-2 i / \sqrt{\pi}$, while another choice, corresponding to the approximant $R_{3,1}$, is given

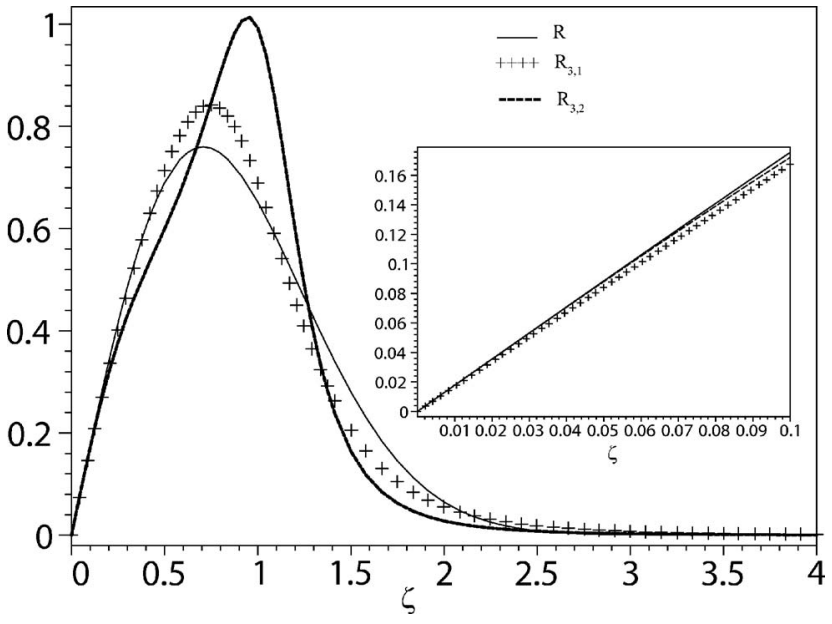

FIG. 1. Comparison between the imaginary part of the function $R(\zeta)$ (thin solid line) with the corresponding Padé approximants $R_{3,1}$ (crosses) and $R_{3,2}$ (thick dashed line). The inset displays the same quantities in a smaller range of $\zeta$, close to the origin.

by $a_{0}=1.03241 i, a_{1}=2.23990$, and $a_{2}=-2.06482 i$. Figure 1 displays a comparison between the imaginary part of the function $R(\zeta)$ (thin solid line) with the Padé approximants $R_{3,2}$ (thick dashed line) and $R_{3,1}$ (crosses). The inset displays the same quantities in a smaller range of $\zeta$, close to the origin. It is conspicuous that $R_{3,2}$ provides a fit that is slightly better for small $\zeta$, but turns out to be globally less accurate than $R_{3,1}$.

\section{Modeling of $\tilde{r}_{\perp \perp}$}

To evaluate $\widetilde{r}_{\perp \perp}$, one starts from the kinetic expression given in (B19) and eliminates the terms involving the plasma response function or the potential $\Phi$. Since $\widetilde{r}_{\perp \perp}$ is negligible at large scales, we can restrict the estimate to the quasitransverse spectral cone and write [see Eq. (42) of Ref. 21]

$$
\left(\frac{T_{\perp}^{(0)}}{T_{\|}^{(0)}} R(\zeta)-1\right) \frac{b_{z}}{B_{0}}=\frac{1}{\Gamma_{0}(b)}\left(\frac{k_{\perp}^{2}}{\Omega} \chi_{s}-\frac{T_{\perp}^{(1)}}{T_{\perp}^{(0)}}\right) .
$$

Here, we have written the proton velocity in the form $\vec{u}_{p}=\vec{u}_{\perp p}+u_{\| p} \hat{z}$ (neglecting, in this linear estimate, the distortion of the magnetic field lines), with a transverse velocity $\vec{u}_{\perp p}=-\nabla \chi_{c}+\nabla \times\left(\chi_{s} \hat{z}\right)$, which gives $k_{\perp}^{2} \chi_{c}=i \vec{k}_{\perp} \cdot \vec{u}_{p}$ and $k_{\perp}^{2} \chi_{s}=\left(i \vec{k}_{\perp} \times \vec{u}_{p}\right) \cdot \hat{z}$. To simplify the writing, we shall in the following use the simpler notation $i \vec{k}_{\perp} \times \vec{u}_{p}$, thus identifying a longitudinal vector with its unique component.

Furthermore, from Eqs. (B1) and (B3), one also has in the quasi-transverse cone

$$
\begin{aligned}
R(\zeta) \frac{e \Psi}{T_{\|}^{(0)}}= & -\frac{n^{(1)}}{n^{(0)}}+\left(\frac{2}{\Gamma_{0}(b)}-1-\frac{\Gamma_{1}(b)}{\Gamma_{0}(b)}\right) \frac{T_{\perp}^{(1)}}{T_{\perp}^{(0)}} \\
& +\left(\frac{1-\Gamma_{0}(b)}{b\left[\Gamma_{0}(b)-\Gamma_{1}(b)\right]}-\frac{2}{\Gamma_{0}(b)}+1\right. \\
& \left.+\frac{\Gamma_{1}(b)}{\Gamma_{0}(b)}\right) \frac{k_{\perp}^{2}}{\Omega} \chi_{s}
\end{aligned}
$$




$$
\begin{aligned}
\frac{e}{T_{\perp}^{(0)}}\left(\Phi+\frac{k_{z}^{2}}{k_{\perp}^{2}}(\Phi-\Psi)\right) \\
=-\frac{n^{(1)}}{n^{(0)}}-\left(1+\frac{\Gamma_{1}(b)}{\Gamma_{0}(b)}\right) \frac{T_{\perp}^{(1)}}{T_{\perp}^{(0)}} \\
+\left(1-\frac{\Gamma_{0}(b)}{b\left[\Gamma_{0}(b)-\Gamma_{1}(b)\right]}+\frac{\Gamma_{1}(b)}{\Gamma_{0}(b)}\right) \frac{k_{\perp}^{2}}{\Omega} \chi_{s} .
\end{aligned}
$$

The expression for $\widetilde{r}_{\perp \perp}$ follows immediately.

\section{MODELING NONGYROTROPIC CONTRIBUTIONS}

\section{A. Nongyrotropic contributions from the fourth-rank cumulants}

Like the other nongyrotropic contributions, the quantities $R_{\|}^{\mathrm{NG}}$ and $R_{\perp}^{\mathrm{NG}}$ entering Eqs. (14) and (15) are evaluated in a linear setting. For this purpose, we replace the other terms arising in these equations by their kinetic expressions given in Appendix B. We get $R_{\|}^{\mathrm{NG}}=0$ and

$$
\begin{aligned}
& \frac{p_{\perp}^{(0)}}{\rho^{(0)}}\left(\nabla_{\perp} \cdot \Pi_{z}\right)+\partial_{z} R_{\perp}^{N G} \\
&=- {\left[1-\Gamma_{0}(b)+2 b \Gamma_{0}(b)-2 b \Gamma_{1}(b)\right] \frac{p_{\perp}^{(0)}}{\rho^{(0)}} } \\
& \times\left(p_{\perp}^{(0)}-p_{\|}^{(0)}\right) \partial_{z} \frac{b_{z}}{B_{0}}-\frac{p_{\perp}^{(0)}}{\rho^{(0)}}\left(p_{\perp}^{(0)}-p_{\|}^{(0)}\right) b \\
& \times\left[\Gamma_{0}(b)-\Gamma_{1}(b)\right] \partial_{z} \frac{e \Psi}{T_{\perp}^{(0)}}+\frac{p_{\perp}^{(0)}}{\rho^{(0)}}\left(p_{\perp}^{(0)}-2 p_{\|}^{(0)}\right) \\
& \times\left[\Gamma_{0}(b)-\Gamma_{1}(b)\right] \frac{c}{\Omega B_{0}}\left[k_{z}(\vec{k} \cdot \vec{E})-\left(k_{\perp}^{2}+k_{z}^{2}\right) E_{z}\right] .
\end{aligned}
$$

Note that this contribution is also relevant for the electrons (with the corresponding definitions of $b$ and $\Omega$, the latter including a negative sign), since the reference to the particle mass drops out in the large-scale limit.

\section{B. Nongyrotropic heat fluxes}

It is convenient to write

$$
\begin{aligned}
& \vec{S}_{\perp}^{\perp}=-\nabla_{\perp} \mathcal{E}+\nabla_{\perp} \times(\mathcal{F} \hat{z}), \\
& \vec{S}_{\perp}^{\|}=-\nabla_{\perp} \mathcal{G}+\nabla_{\perp} \times(\mathcal{H} \hat{z}) .
\end{aligned}
$$

Since only $\nabla \cdot \vec{S}_{\perp}^{\perp}$ and $\nabla \cdot \vec{S}_{\perp}^{\|}$are needed in the equations for the parallel and perpendicular pressures, it is enough to evaluate $\mathcal{E}$ and $\mathcal{G}$.

The quantity $\mathcal{E}$ identifies with the coefficient of $\cos \psi$ in the right-hand side (RHS) of Eq. (B10) for $S_{x}^{\perp}$. It is conveniently rewritten in the form

$$
\begin{aligned}
\mathcal{E}= & -i \frac{p_{\perp}^{(0)} \omega}{k_{\perp}^{2}}\left[2 b \Gamma_{0}(b)-2 b \Gamma_{1}(b)-\Gamma_{1}(b)\right] \frac{b_{z}}{B_{0}} \\
& +p_{\perp}^{(0)} \frac{k_{z}^{2}}{k_{\perp} \omega}\left[-1+\Gamma_{0}(b)+b \Gamma_{0}(b)\right. \\
& \left.-b \Gamma_{1}(b)\right] \frac{e}{T_{\perp}^{(0)}}\left[\frac{T_{\perp}^{(0)}-T_{\|}^{(0)}}{m}\left(1+\frac{k_{z}^{2}}{k_{\perp}^{2}}\right)(\Phi-\Psi)\right. \\
& \left.-\frac{\omega^{2}}{k_{z}^{2}}\left(\Phi+\frac{k_{z}^{2}}{k_{\perp}^{2}} \Phi-\Psi\right)\right] .
\end{aligned}
$$

Using Eq. (B4) to express the quantity inside the square parentheses, one gets

$$
\begin{aligned}
\frac{\mathcal{E}}{p_{\perp}^{(0)}}= & \frac{-i \omega}{k_{\perp}^{2}}\left[\left[2 b \Gamma_{0}(b)-2 b \Gamma_{1}(b)-\Gamma_{1}(b)\right]\right. \\
& \left.+\frac{\left[1-\Gamma_{0}(b)-b \Gamma_{0}(b)+b \Gamma_{1}(b)\right]\left[\Gamma_{0}(b)-\Gamma_{1}(b)\right]}{1-\Gamma_{0}(b)}\right] \frac{b_{z}}{B_{0}} \\
& +\frac{r_{L}^{2}}{2}\left[\frac{-1+\Gamma_{0}(b)+b \Gamma_{0}(b)-b \Gamma_{1}(b)}{b\left[1-\Gamma_{0}(b)\right]}\right]\left(i \vec{k}_{\perp} \cdot \vec{u}_{\perp}\right),
\end{aligned}
$$

where, as already mentioned, $\omega b_{z}=c \vec{k}_{\perp} \times \vec{E}_{\perp}$.

Similarly, $\mathcal{G}$ identifies with the coefficient of $\cos \psi$ in the RHS of Eq. (B11) for $S_{x}^{\|}$, multiplied by the factor $i / k_{\perp}$. It is conveniently rewritten

$$
\begin{aligned}
\mathcal{G}= & \frac{2 i p_{\|}^{(0)}}{k_{\perp}} \frac{k_{z}}{\Omega} \frac{T_{\perp}^{(0)}-T_{\|}^{(0)}}{m} \frac{1-\Gamma_{0}(b)}{b} \frac{4 \pi j_{z}}{c k_{\perp} B_{0}} \\
= & -\frac{2}{\Omega} \frac{T_{\|}^{(0)}}{m}\left(p_{\perp}^{(0)}-p_{\|}^{(0)}\right)\left(\frac{1-\Gamma_{0}(b)}{b}\right) \\
& \times \frac{1}{k_{\perp}^{2} B_{0}} i \vec{k}_{\perp} \cdot\left(i k_{z} \hat{z} \times \vec{b}_{\perp}\right) .
\end{aligned}
$$

Note that $\mathcal{G}$ contributes also for the electrons (with a negative sign originating from the gyrofrequency).

\section{Gyroviscous tensor}

It is convenient to express the gyroviscous force $\nabla_{\perp} \cdot \boldsymbol{\Pi}_{\perp}$ in the form

$$
\frac{1}{p_{\perp p}^{(0)}} \nabla_{\perp} \cdot \Pi_{\perp}=-\nabla_{\perp} \mathcal{A}+\nabla_{\perp} \times(\mathcal{B} \hat{z})
$$

where $-\mathcal{A}$ identifies with the coefficient of $\cos 2 \psi$ and $\mathcal{B}$ with that of $\sin 2 \psi$ in the expression (B8) of $\Pi_{x x} / p_{\perp}^{(0)}$.

In order to get rid of the plasma response function that enters this expression for $\Pi_{x x}$, we use the transverse velocity and the transverse temperature of the ions, as given in Appendix B, and write ${ }^{21}$

$$
\mathcal{A}=\left[1-\frac{\Gamma_{1}(b)}{b\left[\Gamma_{0}(b)-\Gamma_{1}(b)\right]}+\frac{\Gamma_{1}(b)}{\Gamma_{0}(b)}\right] \frac{k_{\perp}^{2}}{\Omega} \chi_{s p}-\frac{\Gamma_{1}(b)}{\Gamma_{0}(b)} \frac{T_{\perp p}^{(1)}}{T_{\perp p}^{(0)}},
$$

where 


$$
\frac{\Gamma_{1}(b)}{\Gamma_{0}(b)} \frac{T_{\perp p}^{(1)}}{T_{\perp p}^{(0)}}=\frac{1}{p_{\perp}^{(0)}} \frac{\Gamma_{1}(b)}{b \Gamma_{0}(b)} n^{(0)} \frac{r_{L}^{2}}{2} k_{\perp}^{2} T_{\perp}
$$

Here, $T_{\perp}$ is the local ion perpendicular temperature. One finally gets

$$
\begin{aligned}
\mathcal{A}= & \frac{1}{\Omega}\left[1-\frac{\Gamma_{1}(b)}{b\left[\Gamma_{0}(b)-\Gamma_{1}(b)\right]}+\frac{\Gamma_{1}(b)}{\Gamma_{0}(b)}\right]\left(\hat{z} \times i \vec{k}_{\perp}\right) \cdot \vec{u}_{\perp} \\
& -\frac{1}{p_{\perp}^{(0)}} \frac{\Gamma_{1}(b)}{b \Gamma_{0}(b)} n^{(0)} \frac{r_{L}^{2}}{2} k_{\perp}^{2} T_{\perp} .
\end{aligned}
$$

Similarly,

$$
\begin{aligned}
\mathcal{B}= & -\frac{i}{\Omega}\left[\frac{\Gamma_{0}(b)-1-\Gamma_{1}(b)}{b}+2\left[\Gamma_{0}(b)-\Gamma_{1}(b)\right]\right. \\
& \left.+\frac{\Gamma_{0}(b)-\Gamma_{1}(b)}{1-\Gamma_{0}(b)}\left(\Gamma_{0}(b)-\Gamma_{1}(b)-\frac{1-\Gamma_{0}(b)}{b}\right)\right] \frac{c}{B_{0}} \\
& \times\left(\vec{k}_{\perp} \times \vec{E}_{\perp}\right)+\frac{1}{1-\Gamma_{0}(b)}\left[\Gamma_{0}(b)-\Gamma_{1}(b)\right. \\
& \left.-\frac{1-\Gamma_{0}(b)}{b}\right] \frac{i}{\Omega} \vec{k}_{\perp} \cdot \vec{u}_{\perp} .
\end{aligned}
$$

Let us now turn to $\vec{\Pi}_{z}=\left(\Pi_{x z}, \Pi_{y z}, \Pi_{z z}\right)$, where $\Pi_{z z}=0$ in the linear description. We write

$$
\vec{\Pi}_{z}=-\nabla_{\perp} \mathcal{C}+\nabla_{\perp} \times(\mathcal{D} \hat{z})
$$

where $-\mathcal{C}$ identifies with the coefficients of $\cos \psi$ and $\mathcal{D}$ with that of $\sin \psi$ in Eq. (B9) for $\Pi_{x z}$, both divided by $i k_{\perp}$. Using Eq. (A5), one has

$$
\begin{aligned}
\frac{\mathcal{C}}{p_{\| p}^{(0)}}= & \frac{i}{k_{\perp}}\left\{\frac { k _ { z } } { k _ { \perp } } ( \frac { T _ { \perp p } ^ { ( 0 ) } } { T _ { \| p } ^ { ( 0 ) } } - 1 ) \left[\left[\Gamma_{0}(b)-\Gamma_{1}(b)-1\right] \frac{b_{z}}{B_{0}}\right.\right. \\
& \left.\left.-\left[1-\Gamma_{0}(b)\right] \frac{e \Psi}{T_{\perp p}^{(0)}}\right]-\frac{\omega}{\Omega}\left(\frac{T_{\perp p}^{(0)}}{T_{\| p}^{(0)}}-2\right) \frac{1-\Gamma_{0}(b)}{b} \frac{4 \pi j_{z}}{c k_{\perp} B_{0}}\right\}
\end{aligned}
$$

that, because of

$$
\omega j_{z}=\frac{i c^{2}}{4 \pi}\left[k_{z}(\vec{k} \cdot \vec{E})-\left(k_{z}^{2}+k_{\perp}^{2}\right) E_{z}\right],
$$

rewrites

$$
\begin{aligned}
\frac{\mathcal{C}}{p_{\| p}^{(0)}}= & -\left(\frac{T_{\perp p}^{(0)}}{T_{\| p}^{(0)}}-1\right) \frac{\Gamma_{0}(b)-\Gamma_{1}(b)-1}{b} \frac{r_{L}^{2}}{2}\left(i \vec{k}_{\perp} \cdot \frac{\vec{b}_{\perp}}{B_{0}}\right) \\
& +\frac{1-\Gamma_{0}(b)}{b} \frac{c}{\Omega} \frac{E_{z}}{B_{0}} \\
& +\frac{1}{\Omega}\left(\frac{T_{\perp p}^{(0)}}{T_{\| p}^{(0)}}-2\right) \frac{1-\Gamma_{0}(b)}{b} \frac{c}{B_{0}} \frac{k_{z}}{k_{\perp}^{2}}\left(\vec{k}_{\perp} \cdot \vec{E}_{\perp}\right) .
\end{aligned}
$$

A more delicate estimate concerns $\mathcal{D}$. Using Eq. (A5), we have

$$
\begin{aligned}
\mathcal{D}= & \frac{p_{\perp p}^{(0)}}{\Omega} \sqrt{\frac{2 T_{\|}^{(0)}}{m}}\left[\Gamma_{0}(b)-\Gamma_{1}(b)\right] \zeta R(\zeta)\left(2 \frac{T_{\perp}^{(0)}}{T_{\|}^{(0)}} \frac{b_{z}}{B_{0}}+\frac{e \Psi}{T_{\|}^{(0)}}\right) \\
& -\left(p_{\perp}^{(0)}-p_{\|}^{(0)}\right)\left[\Gamma_{0}(b)-\Gamma_{1}(b)-1\right] \frac{4 \pi j_{z}}{c k_{\perp}^{2} B_{0}} \\
& +\left(p_{\perp}^{(0)}-2 p_{\|}^{(0)}\right) \frac{k_{z}}{k_{\perp}} \frac{\omega}{\Omega} \frac{b_{z}}{B_{0}}
\end{aligned}
$$

To estimate the first term, we consider $q_{\perp}$ and $u_{z}$, as given by the kinetic theory. We write

$$
\begin{aligned}
\frac{q_{\perp}}{p_{\perp}^{(0)}}+ & u_{z}+\frac{T_{\perp}^{(0)}-T_{\|}^{(0)}}{m}\left(\Gamma_{0}(b)-\Gamma_{1}(b)\right. \\
- & \left.\frac{1-\Gamma_{0}(b)}{b}\right) \frac{k_{\perp}}{\Omega} \frac{4 \pi j_{z}}{c k_{\perp} B_{0}} \\
= & \sqrt{\frac{2 T_{\|}^{(0)}}{m}}\left[\left(b \Gamma_{0}(b)-b \Gamma_{1}(b)-\Gamma_{0}(b)\right.\right. \\
& \left.+\frac{1}{2} \Gamma_{1}(b)\right) \zeta R(\zeta)\left(\frac{2 T_{\perp}^{(0)}}{T_{\|}^{(0)}} \frac{b_{z}}{B_{0}}+\frac{e \Psi}{T_{\|}^{(0)}}\right) \\
& \left.-\frac{1}{2} \Gamma_{1}(b) \zeta R(\zeta) \frac{e \Psi}{T_{\perp}^{(0)}}\right],
\end{aligned}
$$

where the last term is only relevant inside the spectral cone. Defining

$$
\delta(b)=\frac{\Gamma_{0}(b)-\Gamma_{1}(b)}{b \Gamma_{0}(b)-b \Gamma_{1}(b)-\Gamma_{0}(b)+\frac{1}{2} \Gamma_{1}(b)},
$$

it follows that

$$
\begin{aligned}
\mathcal{D}= & \frac{\delta(b)}{\Omega}\left(q_{\perp}+p_{\perp}^{(0)} u_{z}+\frac{1}{2} p_{\|}^{(0)} \sqrt{\frac{2 T_{\|}^{(0)}}{m}} \Gamma_{1}(b) \zeta R(\zeta) \frac{e \Psi}{T_{\|}^{(0)}}\right) \\
& +\left(p_{\perp}^{(0)}-p_{\|}^{(0)}\right)\left\{\delta(b)\left[b \Gamma_{0}(b)-b \Gamma_{1}(b)-\Gamma_{0}(b)+1\right]\right. \\
& \left.-\Gamma_{0}(b)+\Gamma_{1}(b)+1\right\} \frac{4 \pi j_{z}}{k_{\perp}^{2} c B_{0}} \\
& -\left(p_{\perp}^{(0)}-2 p_{\|}^{(0)}\right) \frac{i k_{z} c}{\Omega k_{\perp}^{2} B_{0}}\left(i \vec{k}_{\perp} \times \vec{E}_{\perp}\right),
\end{aligned}
$$

where it is convenient to isolate the heat flux contribution by writing

$$
\mathcal{D}=\frac{\delta(b)}{\Omega} q_{\perp}+D_{1} .
$$

Two comments are then in order. No simple expression in terms of hydrodynamic quantities is available for the contribution $\zeta R(\zeta) \Psi$ arising in $D_{1}$, a point we shall address later on. Furthermore, a special problem concerns the determination of the heat flux $q_{\perp}$ in a way suitable for the slow dynamics regime, where FLR corrections play a central role. In this regime, the heat flux $q_{\perp}$ is not prescribed by its dynamical equation but rather by a quasi-equilibrium condition in the transverse pressure ${ }^{22}$ or temperature equation that, when linearized, reads 


$$
\partial_{t} \frac{T_{\perp}}{T_{\perp}^{(0)}}=-\nabla_{\perp} \cdot \vec{u}_{\perp}-\frac{1}{p_{\perp}^{(0)}}\left(\partial_{z} q_{\perp}+\nabla_{\perp} \cdot \vec{S}_{\perp}^{\perp}\right) .
$$

It is convenient to rewrite this equation in the form

$$
\partial_{z} q_{\perp}=Q_{1}-p_{\perp}^{(0)} \partial_{t} \frac{T_{\perp}^{(1)}}{T_{\perp}^{(0)}}
$$

with $Q_{1}=-\left(p_{\perp}^{(0)} \nabla_{\perp} \cdot \vec{u}_{\perp}+\nabla_{\perp} \cdot \vec{S}_{\perp}^{\perp}\right)$. The last term in the RHS of Eq. (48) is estimated from the kinetic theory that gives

$$
\frac{T_{\perp}^{(1)}}{T_{\perp}^{(0)}}=\frac{1}{\Omega}\left(i \vec{k}_{\perp} \times \vec{u}_{\perp}\right)+\Gamma_{0}(b) \frac{b_{z}}{B_{0}}-\Gamma_{0}(b) \frac{T_{\perp}^{(0)}}{T_{\|}^{(0)}} R(\zeta) \frac{b_{z}}{B_{0}} .
$$

We then approximate the plasma response function $R$ by the one-pole approximant $R_{1}=1 /(1-i \sqrt{\pi \zeta})$ (using a two-pole approximant would also be possible, although more cumbersome). Coming back to the physical space relative to the time variable (without changing the notations), Eq. (49) becomes

$$
\begin{aligned}
\Gamma_{0}(b) \frac{T_{\perp}^{(0)}}{T_{\|}^{(0)}} \frac{b_{z}}{B_{0}}= & \left(1+\sqrt{\pi}\left|k_{z}\right|^{-1} \sqrt{\frac{m}{2 T_{\|}^{(0)}}} \partial_{t}\right) \\
& \times\left[\frac{1}{\Omega}\left(i \vec{k}_{\perp} \times \vec{u}_{\perp}\right)+\Gamma_{0}(b) \frac{b_{z}}{B_{0}}-\frac{T_{\perp}^{(1)}}{T_{\perp}^{(0)}}\right] \\
= & {\left[\frac{1}{\Omega}\left(i \vec{k}_{\perp} \times \vec{u}_{\perp}\right)+\Gamma_{0}(b) \frac{b_{z}}{B_{0}}-\frac{T_{\perp}^{(1)}}{T_{\perp}^{(0)}}\right] } \\
& +\sqrt{\pi}\left|k_{z}\right|^{-1} \sqrt{\frac{m}{2 T_{\|}^{(0)}}}\left[\frac{1}{\Omega} \partial_{t}\left(i \vec{k}_{\perp} \times \vec{u}_{\perp}\right)\right. \\
& \left.-c \Gamma_{0}(b)\left(i \vec{k}_{\perp} \times \frac{\vec{E}_{\perp}}{B_{0}}\right)-\partial_{t} \frac{T_{\perp}^{(1)}}{T_{\perp}^{(0)}}\right] .
\end{aligned}
$$

It follows that

$$
\partial_{t} \frac{T_{\perp}^{(1)}}{T_{\perp}^{(0)}}=\frac{1}{\Omega} \partial_{t}\left(i \vec{k}_{\perp} \times \vec{u}_{\perp}\right)+F_{1},
$$

with

$$
\begin{aligned}
F_{1}= & -c \Gamma_{0}(b)\left(i \vec{k}_{\perp} \times \frac{\vec{E}_{\perp}}{B_{0}}\right)+\frac{1}{\sqrt{\pi}} \sqrt{\frac{2 T_{\|}^{(0)}}{m}}\left|k_{z}\right|\left[-\frac{T_{\perp}^{(1)}}{T_{\perp}^{(0)}}\right. \\
& \left.+\frac{1}{\Omega}\left(i \vec{k}_{\perp} \times \vec{u}_{\perp}\right)-\Gamma_{0}(b)\left(\frac{T_{\perp}^{(0)}}{T_{\|}^{(0)}}-1\right) \frac{b_{z}}{B_{0}}\right] .
\end{aligned}
$$

This leads to

$$
\mathcal{D}=-\frac{\delta(b)}{i k_{z}} \frac{p_{\perp}^{(0)}}{\Omega^{2}} \partial_{t}\left(i \vec{k}_{\perp} \times \vec{u}_{\perp}\right)+D_{2},
$$

with

$$
D_{2}=D_{1}+\frac{1}{\Omega} \frac{\delta(b)}{i k_{z}}\left(Q_{1}-p_{\perp}^{(0)} F_{1}\right) .
$$

Furthermore, from the linearized equation for transverse velocity, we get

$$
\partial_{t}\left(i \vec{k}_{\perp} \times \vec{u}_{\perp}\right)=-k_{\perp}^{2} \frac{1}{\rho^{(0)}} i k_{z} \mathcal{D}+U_{1},
$$

with

$$
U_{1}=\left(c_{A}^{2}+\frac{p_{\perp T}^{(0)}-p_{\| T}^{(0)}}{\rho^{(0)}}\right)\left(i k_{z}\right)\left(i k_{x} \frac{b_{y}}{B_{0}}-i k_{y} \frac{b_{x}}{B_{0}}\right)-k_{\perp}^{2} \frac{p_{\perp}^{(0)}}{\rho^{(0)}} \mathcal{B},
$$

where $p_{\perp T}^{(0)}$ and $p_{\| T}^{(0)}$ denote the total (ion+electron) perpendicular and parallel pressures. Substituting Eq. (55) into Eq (53), we get

$$
[1-b \delta(b)] \mathcal{D}=D_{3},
$$

where

$$
D_{3}=D_{2}-\frac{\delta(b)}{i k_{z}} \frac{p_{\perp}^{(0)}}{\Omega^{2}} U_{1} .
$$

One easily checks that the coefficient of $\mathcal{D}$ in the LHS of Eq. (57) does not vanish.

We now have to estimate $\zeta R(\zeta) e \Psi / T_{\|}^{(0)}$ that enters $D_{1}$. When, consistent with the previous approximation, $R$ is replaced by $R_{1}$, one simply has $\zeta R_{1}(\zeta)=(i / \sqrt{\pi})\left[1-R_{1}(\zeta)\right]$. We then use Eq. (25) that expresses $R(\zeta) e \Psi / T_{\|}^{(0)}$ in terms of hydrodynamic quantities. Note that the approximation of the plasma response function by its one-pole approximant $R_{1}$ (whose large- $\zeta$ behavior is inaccurate) is acceptable in terms that are mostly relevant for the slow dynamics. Indeed, $\zeta \ll 1$ for mirror waves and is of order unity for kinetic Alfvén waves (KAWs).

We conclude this section by stressing the importance of the above modeling of the heat flux $q_{\perp}$ in the estimate of a gyroviscous stress tensor that, as already mentioned, is mostly relevant for the slow dynamics: using $q_{\perp}$ as given by the heat flux dynamical equation (a typically fast dynamics estimate) indeed leads to a spurious instability for a non propagating normal mode, at the scale of the ion gyroradius.

\section{DISPERSION AND DAMPING OF MHD WAVES}

In order to evaluate the accuracy of the above model, we compare in this section the frequency and collisionless damping rate of various MHD waves, as obtained by solving the dispersion relation of the resulting linearized system, with the predictions of the full linear kinetic theory, as given by the WHAMP program. ${ }^{23,28}$ Plasma parameters of different magnitudes and various directions of propagation are considered.

\section{A. Kinetic Alvén waves}

Figure 2 displays the frequency and damping rate, both normalized by $k_{\|} v_{\mathrm{A}}$, as a function of $k_{\perp} r_{\mathrm{L}}$ for kinetic Alfvén waves propagating in a quasi-transverse direction characterized by an angle $\theta \equiv \tan ^{-1}\left(k_{\perp} / k_{\|}\right)=\tan ^{-1}(1000)$, in a plasma with isotropic temperatures such that $\tau \equiv T_{e}^{(0)} / T_{i}^{(0)}=10^{-2}$. Three values, i.e., $\beta=0.1,1$, and 10 , are considered. The solid line is the full kinetic theory, and the crosses refer to predictions of the Landau fluid model, using the three-pole Padé approximant $R_{3,2}(\zeta)$. The crosses in the top panels cor- 

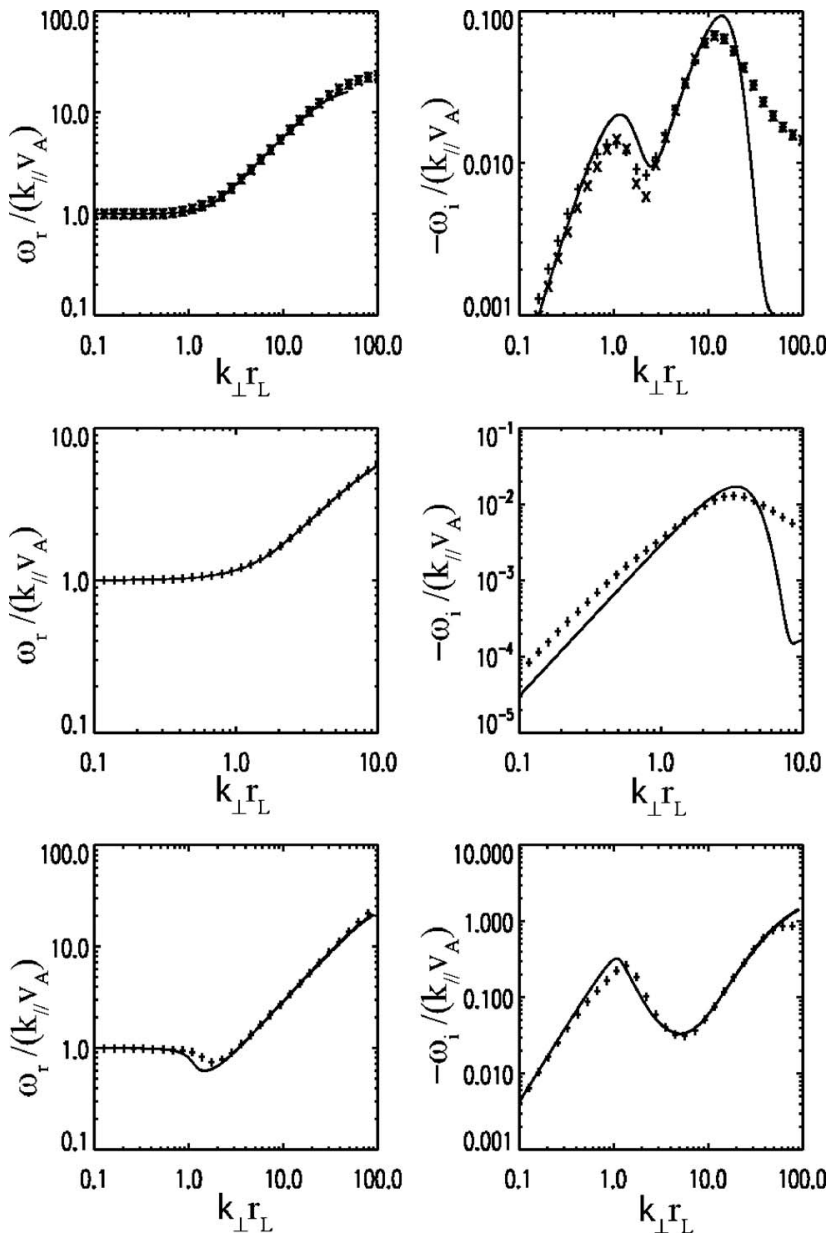

FIG. 2. Normalized frequency $\omega_{r} /\left(k_{\|} v_{\mathrm{A}}\right)$ (left) and damping rate $-\omega_{i} /\left(k_{\|} v_{\mathrm{A}}\right)$ (right) vs $k_{\perp} r_{\mathrm{L}}$, for KAWs with $\theta=\tan ^{-1}(1000), \tau=10^{-2}$, and $\beta=1$ (top), $\beta$ $=0.1$ (middle), and $\beta=10$ (bottom). Here and in the following figures, the solid line refer to the full kinetic theory and the crosses to the prediction of the model when using the $R_{2,3}$ Padé approximant; The crosses in the top panels are obtained with the $R_{3,1}$ Padé approximant.

respond to the Padé approximant $R_{3,1}(\zeta)$ (see the discussion in Sec. III). Similar to what was observed in the comparison of the imaginary part of the plasma response function in Fig. 1 , we observe that the former choice provides a fit that turns out to be globally more accurate, while the latter is slightly better at large scales. In the forthcoming comparisons, we thus retain the former choice. Inspection of the graphs shows that when increasing $\beta$, the validity range of the model extends to a smaller fraction of the ion Larmor radius, but remains essentially unchanged when the spatial scale is expressed in terms of the ion inertial length $v_{\mathrm{A}} / \Omega_{p}$.

Figure 3 is still concerned with quasi-transverse propagating KAWs with $\theta=\tan ^{-1}(1000)$, for $\beta=0.1,1$, and 10 , but in the case of equal ion and electron temperatures $(\tau=1)$. In spite of a much larger damping rate, the agreement with the kinetic theory remains very satisfactory. The values of the parameters used in Figs. 2 and 3 (and also the normalization of the frequency and of the decay rate) were chosen to make easy comparisons with computations based on the gyrokinetic theory ${ }^{9}$ whose accuracy for this extreme propagation angle is almost perfect. Although less accurate, the FLRLandau fluid appears to be able to address a similar regime.
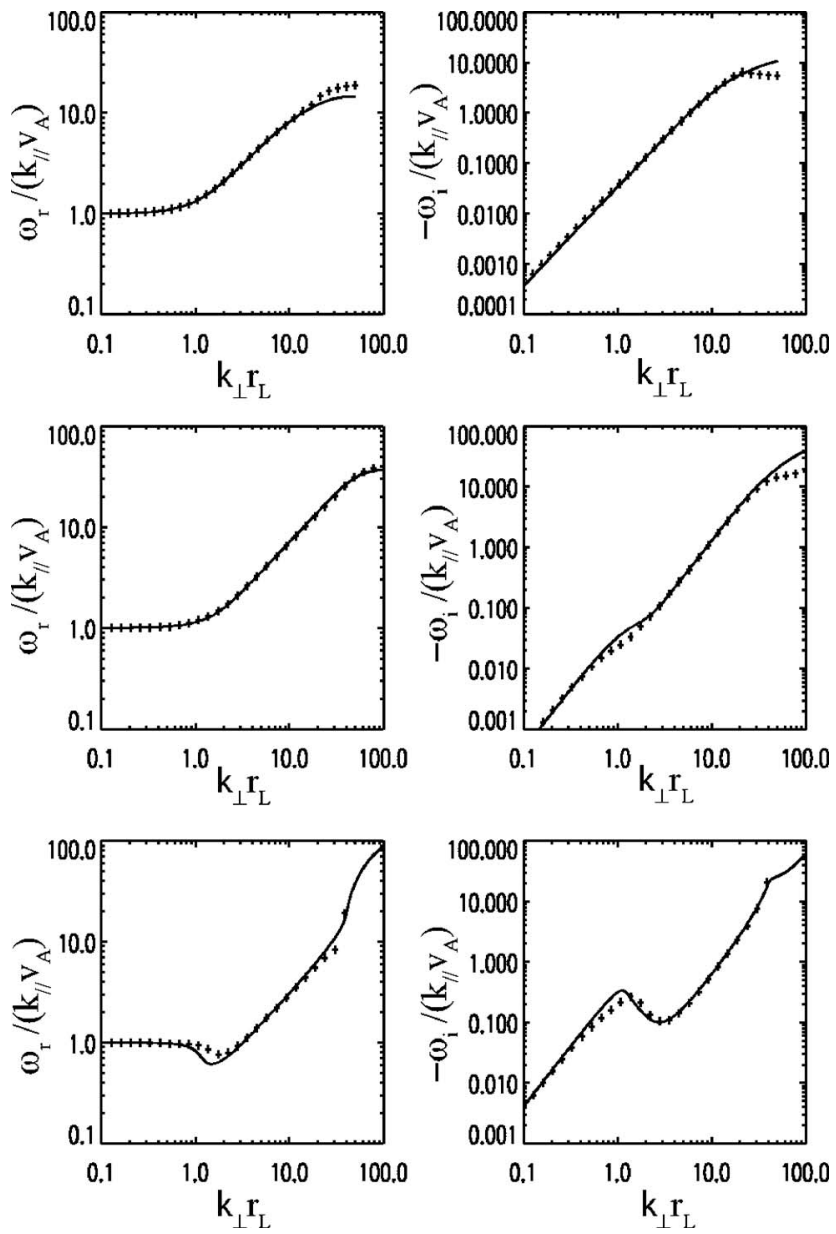

FIG. 3. Normalized frequency $\omega_{r} /\left(k_{\|} v_{\mathrm{A}}\right)$ (left) and damping rate $-\omega_{i} /\left(k_{\|} v_{\mathrm{A}}\right)$ (right) vs $k_{\perp} r_{\mathrm{L}}$, for KAWs with $\theta=\tan ^{-1}(1000), \tau=1$, and for $\beta=0.1$ (top), $\beta=1$ (middle), and $\beta=10$ (bottom).

The main source of error could be linked to the replacement of the plasma response function by a Padé approximant that introduces a sizable discrepancy for modes with parallel phase velocity comparable to the ion thermal velocity.

As in gyrokinetic theory, the present model can capture the small-scale dynamics in quasi-transverse directions only, and in particular misses the ion cyclotron resonance. In order to estimate the spectral range of accuracy of the model when the angle with the ambient field is reduced, we fix $\beta=1$ and $\tau=0.01$ and display in Fig. 4 the cases $\theta=\tan ^{-1}(10) \approx 84.3^{\circ}$ and $\theta=60^{\circ}$. For the former angle, the kinetic theory suggests the existence of a resonance for $k_{\perp} r_{\mathrm{L}} \approx 3$ that is not reproduced by the model, leading to a large error on the decay rate at smaller scales. At larger scales, the agreement is in contrast excellent. For $\theta=60^{\circ}$, we note a difference by a factor of 2 on the decay rate, even for $k_{\perp} r_{\mathrm{L}}<1$. Furthermore, as expected, the strong ion cyclotron damping that takes place at small scale is not reproduced.

In order to estimate the validity range in the case of quasi-parallel Alfvén waves, we consider in Fig. 5 a propagation angle $\theta=\tan ^{-1}(0.1)$ in a plasma with equal ion and electrons temperatures $(\tau=1)$ and $\beta=0.1$ or $\beta=1$. In such a regime, frequency and decay rate are plotted versus $k r_{L}$. In both cases, the frequency is satisfactorily reproduced up to 

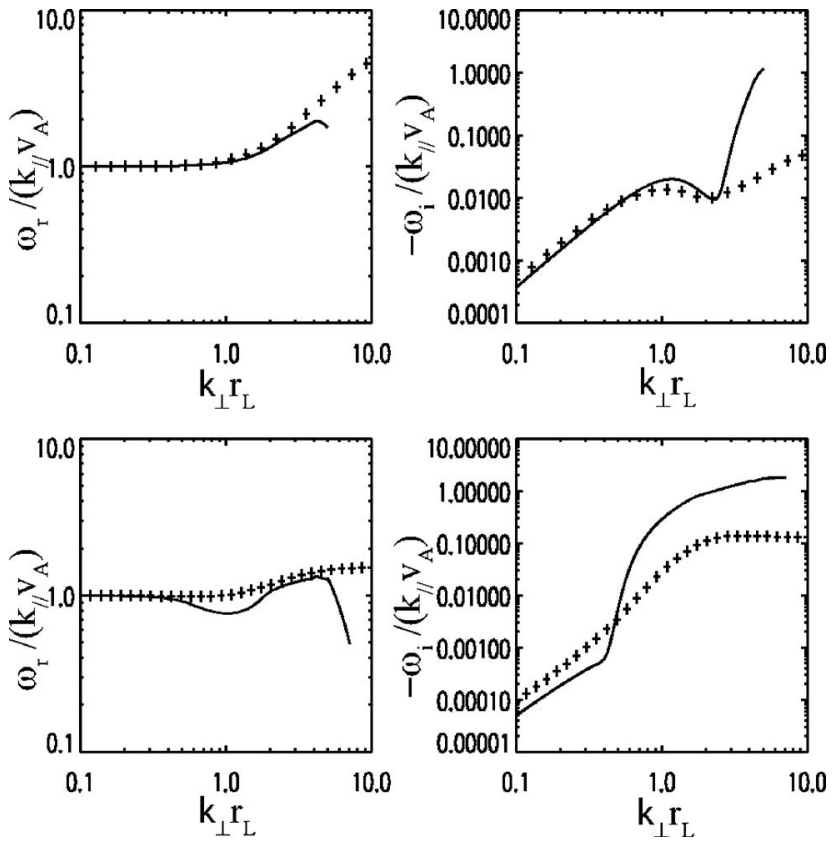

FIG. 4. Normalized frequency $\omega_{r} /\left(k_{\|} v_{\mathrm{A}}\right)$ (left) and damping rate $-\omega_{i} /\left(k_{\|} v_{\mathrm{A}}\right)$ (right) vs $k_{\perp} r_{\mathrm{L}}$, for KAWs with $\tau=0.01, \beta=1$ for $\theta=\tan ^{-1}(10)$ (top) and $\theta=60^{\circ}$ (bottom).

scales comparable to a fraction of ion Larmor radius. In contrast, the model does not reproduce the sharp increase of dissipation rate that takes place near $k r_{\mathrm{L}}=0.1$, again under the effect of the ion cyclotron resonance.

\section{B. Slow magnetosonic waves}

Restricting the comparison to a quasi-transverse propagation $\left[\theta=\tan ^{-1}(1000)\right]$, we consider in Fig. 6 a situation close to the fluid regime characterized by electrons much
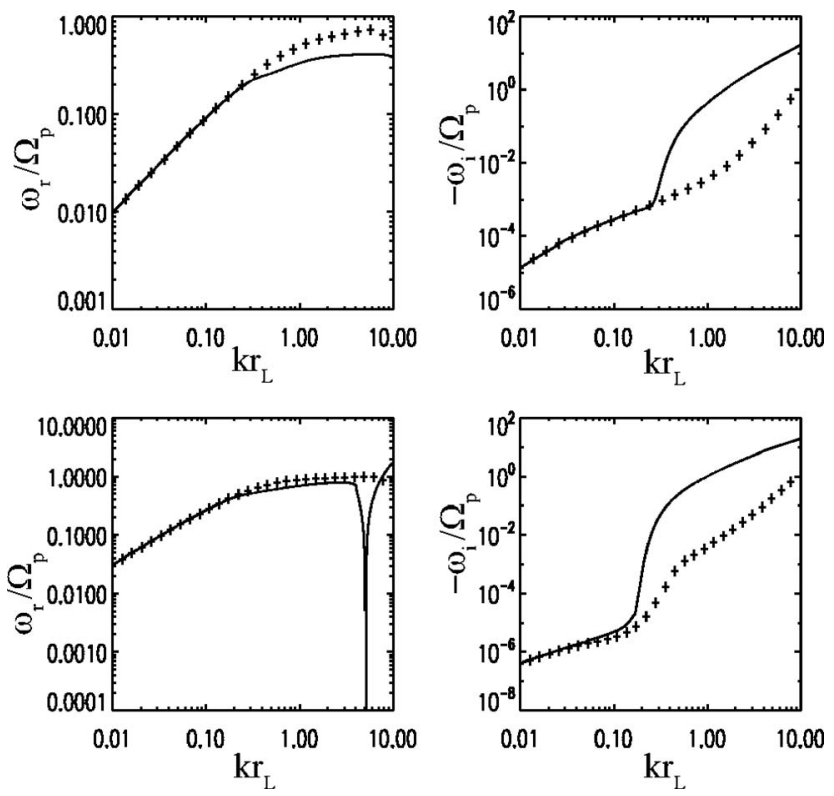

FIG. 5. Normalized frequency $\omega_{r} /\left(k_{\|} v_{\mathrm{A}}\right)$ (left) and damping rate $-\omega_{i} /\left(k_{\|} v_{\mathrm{A}}\right)$ (right) vs $k r_{\mathrm{L}}$, for AWs with $\tau=1, \theta=\tan ^{-1}(0.1)$, and $\beta=1$ (top) and $\beta=0.1$ (bottom).
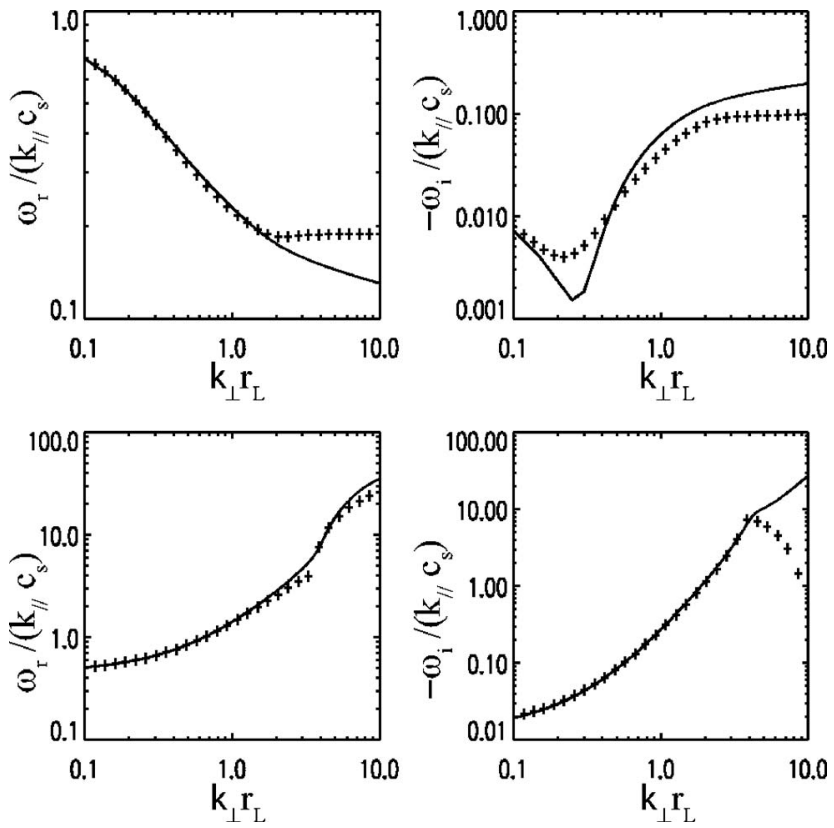

FIG. 6. Normalized frequency $\omega_{r} /\left(k_{\|} c_{s}\right)$ (left) and damping rate $-\omega_{i} /\left(k_{\|} c_{s}\right)$ (right), where $c_{s}$ is the sound velocity, vs $k_{\perp} r_{\mathrm{L}}$, for quasi-transverse slow waves $\left(\theta=\tan ^{-1}(1000)\right)$ with $\tau=100$, and $\beta=0.01$ (top) and $\beta=0.1$ (bottom).

hotter than ions $(\tau=100)$, with $\beta=0.01$ and $\beta=0.1$. In this case, the frequency unit is taken as $k_{\|} c_{s}$, where $c_{s}=\left(T_{e} / m_{p}\right)^{1 / 2}$. We note for the latter value of $\beta$, a satisfactory agreement up to $k_{\perp} r_{\mathrm{L}}$ of a few units, but this extension is more limited when $\beta$ is smaller. In order to address the effect of varying the ion to electron temperature ratio, we consider in Fig. 7 quasi-transverse waves with $\beta=10^{-2}$, for $\tau=10$ and $\tau=1$. In the former case, a qualitative agreement is obtained, in spite of a conspicuous discrepancy of the decay
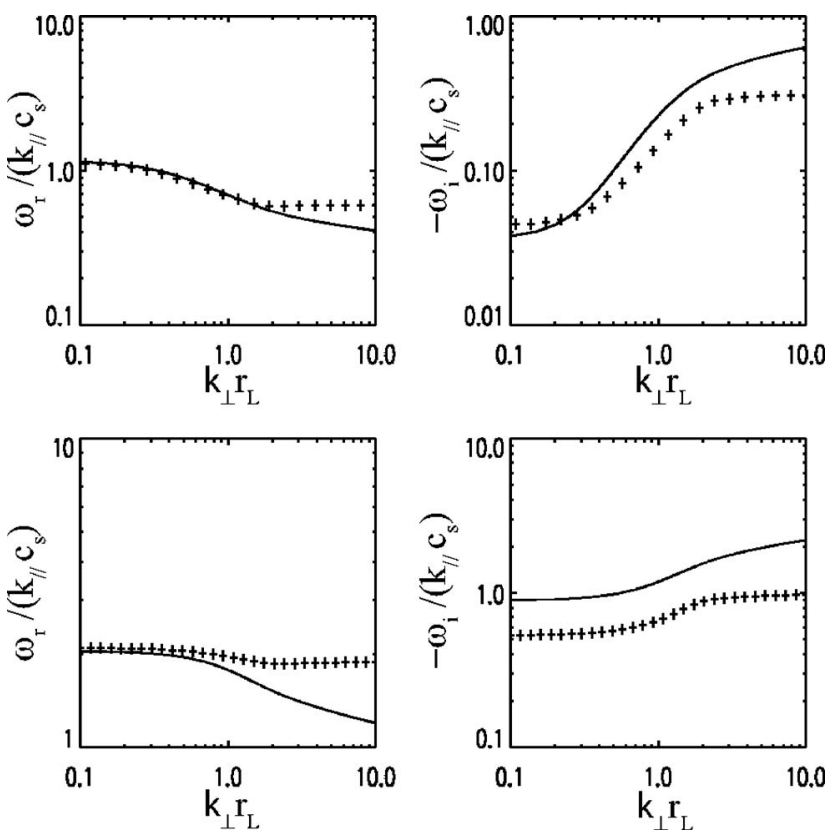

FIG. 7. Normalized frequency $\omega_{r} /\left(k_{\|} c_{s}\right)$ (left) and damping rate $-\omega_{i} /\left(k_{\|} c_{s}\right)$ (right) vs $k_{\perp} r_{\mathrm{L}}$ for quasi-transverse slow waves $\left(\theta=\tan ^{-1}(1000)\right)$ with $\beta=0.01$, and $\tau=10$ (top) and $\tau=1$ (bottom). 

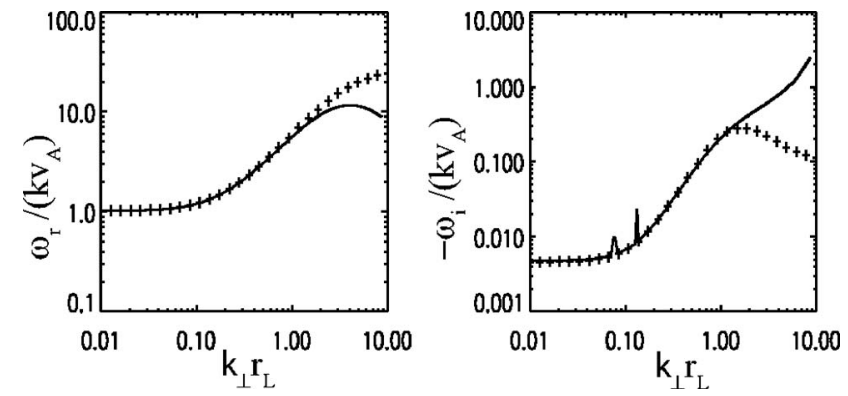

FIG. 8. Normalized frequency $\omega_{r} /\left(k v_{\mathrm{A}}\right)$ (left) and damping rate $-\omega_{i} /\left(k v_{\mathrm{A}}\right)$ (right) vs $k_{\perp} r_{\mathrm{L}}$ for fast waves with $\theta=60, \tau=10$, and $\beta=0.01$.

rate even at large scales. For equal ion and electron temperatures, the damping rate is strongly underestimated at all scales. Indeed, decreasing the parameter $\tau$ strongly enhances the collisionless decay rate, making its value inconsistent with the low-frequency asymptotics.

\section{Fast magnetosonic waves}

It is also of interest to consider the fast magnetosonic waves that are scaled out in the gyrokinetic approach. In the quasi-transverse case $\theta=\tan ^{-1}(1000)$, with $\beta=0.01$ and $\tau=10$ (not shown), Landau damping is, as expected, essentially zero. The negative sign of the dispersion contribution to the frequency, seen in the linear kinetic theory for scales smaller than $k_{\perp} r_{\mathrm{L}}=1$, is not reproduced. Capturing this effect, would indeed require an estimate of the FLR corrections with a $(\omega / \Omega)^{2}$ accuracy. ${ }^{17}$ For oblique propagation $\left(\theta=60^{\circ}\right)$ with the same values of $\beta$ and $\tau$ as above, a high accuracy is obtained up to about $k_{\perp} r_{L}=2$ (Fig. 8). Quasiparallel propagation $\left[\theta=\tan ^{-1}(0.1)\right]$ is considered with $\tau=1$ in Fig. 9 for $\beta=0.1,1$, and 10. For the lowest value of $\beta$, an excellent agreement is obtained up to $k_{\perp} r_{L}=10$, due to the least importance of FLR terms and the absence of ion cyclotron resonance up to these scales. For the intermediate value, a deviation between the model and the kinetic theory becomes visible near $k r_{\mathrm{L}} \approx 1$, but a qualitative agreement is preserved up to $k r_{\mathrm{L}} \approx 10$. For $\beta=10$, WHAMP predictions display a resonance near $k r_{\mathrm{L}}=0.3$, beyond which one observes a strong discrepancy with the model. Although the growth of the dissipation rate is preserved, it is significantly weaker than predicted by the kinetic theory.

\section{Mirror instability}

The mirror instability provides a framework to validate the model in a regime where the temperature anisotropies play an important role. The instability growth rate (normalized by the ion gyrofrequency $\Omega_{p}$ ) that we compare with the linear kinetic theory, results from a short-time evolution of the (nonlinear) FLR-Landau fluid equations, starting with a weak perturbation of the trivial equilibrium. Figure 10 (top, left) corresponds to a simulation very close to the instability threshold, in the case of a propagation angle $\theta=\cos ^{-1}(0.1)$, with $T_{\| e} / T_{\| p}=0.1, T_{p \perp} / T_{p \|}=1.2$ and isotropic electron temperature $\left(T_{e \perp} / T_{e \|}=1\right)$. An excellent agreement is observed, including the quenching of the instability at small scale. A
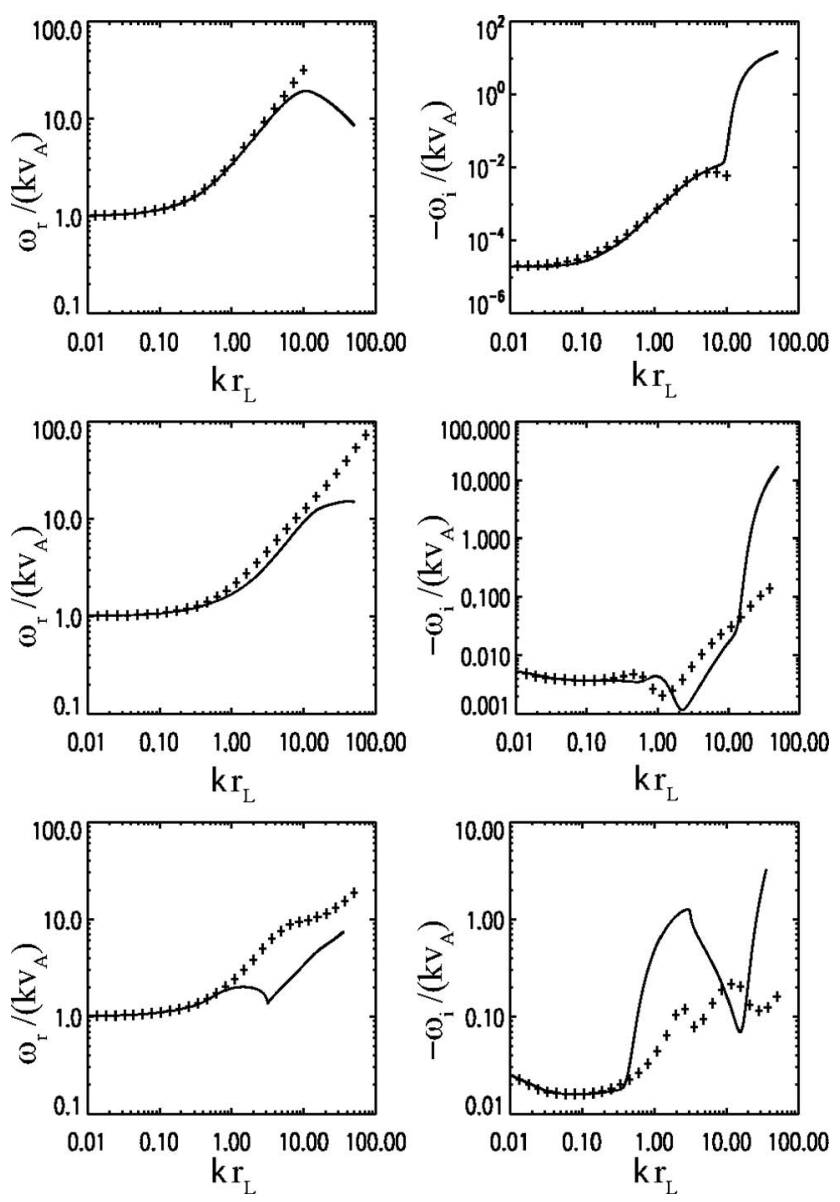

FIG. 9. Normalized frequency $\omega_{r} /\left(k v_{A}\right)$ (left) and damping rate $-\omega_{i} /\left(k v_{\mathrm{A}}\right)$ (right) vs $k r_{\mathrm{L}}$, for fast waves with $\theta=\tan ^{-1}(0.1), \tau=1$, and $\beta=0.1$ (top), $\beta=1$ (middle), and $\beta=10$ (bottom).

simulation at larger distance from threshold is shown in the top-right panel, where $\beta_{p \|}=2$ and $T_{p \perp} / T_{p \|}=2$. The agreement remains very good at large and small scales but a sizable inaccuracy is observed for the most unstable scales. A smaller angle of propagation $\left[\theta=\cos ^{-1}(0.2)\right]$ is displayed in the case $\beta_{p \|}=5$, assuming $T_{p \perp} / T_{p \|}=1.4$ and isotropic electron temperature (bottom, left). Finally, an anisotropic electron temperature $\left(T_{e \perp} / T_{e \|}=1.18\right)$ is considered for $T_{p \perp} / T_{p \|}$ $=1.1$ (bottom right). Increasing slightly further the electron anisotropy to 1.19 shifts dramatically the most unstable scale to $k_{\perp} r_{\mathrm{L}}$ close to 8 (not shown).

\section{CONCLUSION}

We have presented a FLR-Landau fluid model that extends anisotropic MHD equations by including a description of Landau damping and FLR corrections based on the linearized Vlasov-Maxwell equations evaluated in the gyrokinetic limit. This model is thus able to reproduce the proper lowfrequency linear physics not only at large scales where it reduces to the Snyder et al. Landau fluid, ${ }^{16}$ supplemented with the large-scale FLR contributions, ${ }^{17}$ but also for quasitransverse scales that can be significantly smaller than the ion gyroradius. Being based on the classical MHD equations, it can capture both the slow and fast dynamics. 

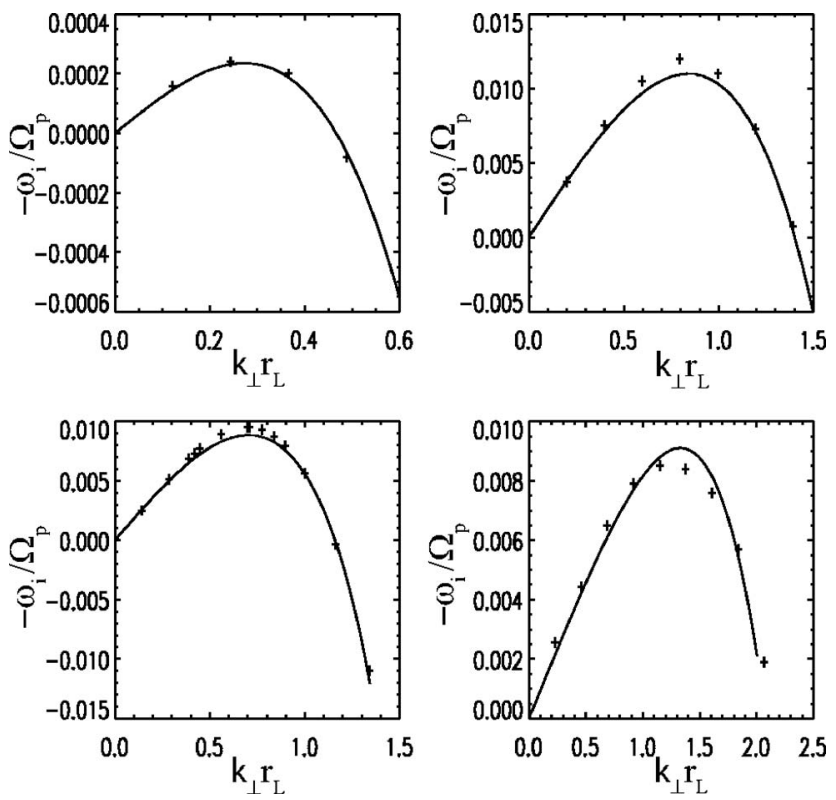

FIG. 10. Normalized growth rate $\omega_{i} / \Omega_{p}$ vs $k_{\perp} r_{\mathrm{L}}$ for mirror modes with $\beta=5, \tau=0.1, \theta=\cos ^{-1}(0.1), T_{\perp p} / T_{\| p}=1.2$, and $T_{\perp e} / T_{\| e}=1$ (top, left) and $\beta=2, \tau=1, \theta=\cos ^{-1}(0.1), T_{\perp p} / T_{\| p}=2$ and $T_{\perp e} / T_{\| e}=1$ (top, right) as a function of $k_{\perp} r_{\mathrm{L}}$. Same for $\beta=5, \quad \tau=1, \quad \theta=\cos ^{-1}(0.2), T_{\perp p} / T_{\| p}=1.4$, and $T_{\perp e} / T_{\| e}=1$ (bottom, left). $T_{\perp p} / T_{\| p}=1.1$ and $T_{\perp e} / T_{\| e}=1.18$ (bottom, right).

Dispersion and collisionless damping of kinetic Alfvén waves are in particular reproduced for a broad range of parameters values. The spectral range that is accurately described becomes more limited as the propagation angle is decreased, but the large-scale dynamics in the oblique and quasi-parallel directions is correctly captured. Fast magnetosonic waves are also retained and correctly described up to scales of the order of the ion Larmor radius, or even smaller for quasi-parallel propagation where FLR corrections are not essential.

By fitting with the nonlinear expressions of the largescale FLR corrections, as derived in the hydrodynamic context, ${ }^{24}$ it is possible to introduce some degree of nonlinearity in the evaluation of the gyroviscous tensor, that takes into account the local variation of the plasma equilibrium quantities, such as the pressures. ${ }^{25}$ On the other hand, the change of reference frame that would be needed to take into account the variation in direction of the local magnetic field, would introduce a prohibitively high level of complexity.

Taking into account possible temperature anisotropies of the equilibrium state, the present model also reproduces the mirror instability growth rate, including its quenching at small scales. However, lacking nonlinear kinetic effects, the model may only provide a qualitative description of phenomena arising at the scale of the ion gyroradius such as the formation of magnetic holes and humps resulting from the mirror mode instability, that was shown to be sensitive to these effects and in particular to the local variation of the ion gyroradius. ${ }^{26}$ Implementing such variations in the present formalism, possibly at a phenomenological level, is under way.

In conclusion, the FLR-Landau fluid model discussed in this paper should be particularly useful for the simulation of turbulence in a strongly magnetized collisionless plasma in a range of scales extending from the hydrodynamic ones to a fraction of the ion Larmor radius. It indeed correctly describes the full dynamics at relatively large scales and also the transfer to the kinetic scales where the noncollisional dissipation of the various MHD modes is accurately reproduced (except its possible arrest under the effect of particle trapping that, as in the gyrofluids, is overlooked by the present description). As already checked in one-dimensional preliminary simulations,${ }^{25}$ no artificial dissipation is needed, provided the wave amplitudes are not too large. This model should thus provide an efficient tool for a direct evaluation of the cascade directions and of the heating rates in collisionless magnetized plasmas, with a relatively modest cost in comparison with fully kinetic simulations. Preliminary numerical investigations in one space dimension have reproduced the existence of inverse cascades resulting from the decay of large-scale KAWs, as predicted by the kinetic theory. ${ }^{27}$

\section{ACKNOWLEDGMENTS}

G. Belmont and L. Rezeau are thanked for their help in using the WHAMP program.

This work benefited of support from CNRS programs "Soleil-Terre," and "Physique et Chimie du Milieu Interstellaire."

\section{APPENDIX A: THE ELECTROMAGNETIC FIELD}

The Vlasov equation is supplemented by the Maxwell equations that express the electric and magnetic fields $\vec{E}$ and $\vec{B}$ in terms of the current $\vec{j}=\Sigma_{r} q_{r} n_{r} \int \vec{v} f_{r} d^{3} v$ and the total charge $\nu=\Sigma_{r} q_{r} n_{r} \int f_{r} d^{3} v$, in the usual form $\partial_{t} \vec{B}=-c \nabla \times \vec{E}$, $c \nabla \times \vec{B}=4 \pi \vec{j}+\partial_{t} \vec{E}$ and $\nabla \cdot \vec{E}=4 \pi \nu$.

It is usual to introduce the scalar potentials $\Phi$ and $\Psi$ together with the vector potential $\vec{A}$ in the form $\vec{E}_{\perp}=-\nabla_{\perp} \Phi$ $-(1 / c) \partial_{t} \vec{A}_{\perp}, E_{z}=-\partial_{z} \Psi$, and $\vec{B}=B_{0} \hat{z}+\nabla \times \vec{A}$, with $\nabla \cdot \vec{A}=0$. It follows that $A_{z}=\left(c k_{z} / \omega\right)(\Phi-\Psi)$, where, since there is no ambiguity, we use the same notation for a field and its Fourier transform.

Assuming a plasma made of protons (subscript $p$ ) and electrons (subscript $e$ ) with charge $q_{p}=-q_{e}=e$, one rewrites the Maxwell-Gauss equation in the nondimensional form:

$$
\frac{c_{\mathrm{A}}^{2}}{c^{2}} b\left(1+\frac{k_{z}^{2}}{k_{\perp}^{2}}\right) \frac{e \Phi}{T_{\perp p}^{(0)}}=\frac{n_{p}}{n^{(0)}}-\frac{n_{e}}{n^{(0)}} .
$$

Here we introduce the standard notation (to be distinguished from the magnetic field components that involve subscripts) $b=T_{\perp p}^{(0)} k_{\perp}^{2} /\left(m_{p} \Omega^{2}\right)$ as a dimensionless measure of the square transverse wavenumber, where $\Omega=e B_{0} /\left(m_{p} c\right)$ denotes the proton gyrofrequency, and $c_{\mathrm{A}}=B_{0} / \sqrt{4 \pi m_{p} n^{(0)}}$ is the Alfvén speed. Assuming $c_{\mathrm{A}} \ll c$ implies local electric neutrality $n_{p}=n_{e}$. Under this assumption, one can also neglect the displacement current and get

$$
k^{2}(\Phi-\Psi)=-\frac{4 \pi}{c^{2}} \frac{\omega}{k_{z}} j_{z} .
$$

The transverse magnetic field components are given by 


$$
\begin{aligned}
& b_{x}=-\frac{k_{x} k_{z}}{k_{\perp}^{2}} b_{z}+i \frac{c}{\omega} k_{y} k_{z}\left(1+\frac{k_{z}^{2}}{k_{\perp}^{2}}\right)(\Phi-\Psi), \\
& b_{y}=-\frac{k_{y} k_{z}}{k_{\perp}^{2}} b_{z}-i \frac{c}{\omega} k_{x} k_{z}\left(1+\frac{k_{z}^{2}}{k_{\perp}^{2}}\right)(\Phi-\Psi) .
\end{aligned}
$$

It follows that in terms of $j_{z}=[c /(4 \pi)]\left(\partial_{x} b_{y}-\partial_{y} b_{x}\right)$,

$$
\frac{k_{z}}{\omega}\left(1+\frac{k_{z}^{2}}{k_{\perp}^{2}}\right) \frac{e}{T_{\perp}^{(0)}}(\Phi-\Psi)=\frac{1}{b} \frac{k_{\perp}}{\Omega} \frac{4 \pi j_{z}}{c k_{\perp} B_{0}} .
$$

\section{APPENDIX B: KINETIC EXPRESSION OF THE FLUID MOMENTS}

We consider the case of linear perturbations in the form of plane waves with a wavevector given by $\left(k_{x}=k_{\perp} \cos \psi, k_{y}=k_{\perp} \sin \psi, k_{z}\right)$. The perturbations of the hydrodynamic moments are easily computed in a lowfrequency expansion, retaining only contributions up to order $\omega / \Omega \sim\left(k_{z} / \Omega\right) \sqrt{2 T_{\|}^{(0)} / m} \ll 1$, with no condition on the transverse scales as measured by $\left(k_{\perp} / \Omega\right) \sqrt{2 T_{\perp}^{(0)} / m}$. Let us also introduce $b=T_{\perp}^{(0)} k_{\perp}^{2} / m \Omega^{2}=k_{\perp}^{2} r_{\mathrm{L}}^{2} / 2$, where $r_{\mathrm{L}}$ is the gyroradius, $\zeta=\left(\omega /\left|k_{z}\right|\right) \sqrt{m / 2 T_{\|}^{(0)}}$, and define the functions $\Gamma_{\nu}(b)=e^{-b} I_{\nu}(b)$ in terms of the modified Bessel function $I_{\nu}(b)$. A standard calculation leads to the following results in terms of the plasma response function $R(\zeta)=1+\zeta Z(\zeta)$, where $Z(\zeta)$ is the plasma dispersion function. We consider explicitly the case of the ions. The corresponding formulas for the electrons are easily obtained, neglecting corrections of the order of the electron-to-proton mass ratio.

\section{Particle density}

Fluctuations of the number density are given by

$$
\begin{aligned}
\frac{n^{(1)}}{n^{(0)}}= & -\left[\Gamma_{0}(b)-\Gamma_{1}(b)\right]\left(\frac{T_{\perp}^{(0)}}{T_{\|}^{(0)}} R(\zeta)-1\right) \frac{b_{z}}{B_{0}} \\
& -\Gamma_{0}(b) R(\zeta) \frac{e \Psi}{T_{\|}^{(0)}}-\frac{e}{T_{\perp}^{(0)}}\left[1-\Gamma_{0}(b)\right] \\
& \times\left(\Phi+\frac{k_{z}^{2}}{k_{\perp}^{2}}(\Phi-\Psi)\right) .
\end{aligned}
$$

\section{Proton velocity}

The transverse ion velocity reads [see Eqs. (12) and (13) of Ref. 21]

$$
\vec{u}_{\perp p}=-\nabla_{\perp} \chi_{c p}+\nabla_{\perp} \times\left(\chi_{s p} \hat{z}\right),
$$

with

$$
\begin{aligned}
\chi_{s p}= & \frac{1}{\Omega} \frac{T_{\perp p}^{(0)}}{m_{p}}\left[\Gamma_{0}(b)-\Gamma_{1}(b)\right]\left[2\left(\frac{T_{\perp p}^{(0)}}{T_{\| p}^{(0)}} R\left(\zeta_{p}\right)-1\right) \frac{b_{z}}{B_{0}}\right. \\
& \left.+R\left(\zeta_{p}\right) \frac{e \Psi}{T_{\| p}^{(0)}}-\frac{e}{T_{\perp p}^{(0)}}\left(\Phi+\frac{k_{z}^{2}}{k_{\perp}^{2}}(\Phi-\Psi)\right)\right] \\
& -4 \frac{T_{\perp p}^{(0)}-T_{\| p}^{(0)}}{m_{p}} \frac{k_{z}^{2}}{k_{\perp}^{2} \Omega} C_{1}^{3}(b) \frac{b_{z}}{B_{0}}
\end{aligned}
$$

where $C_{1}^{3}(b)$, evaluated in Ref. 21 is a function of $b$ only, which has a limit of $1 / 4$ as $b \rightarrow 0$. Furthermore,

$$
\begin{aligned}
\chi_{c p}= & \frac{i \omega}{k_{\perp}^{2}}\left[\Gamma_{0}(b)-\Gamma_{1}(b)\right] \frac{b_{z}}{B_{0}}+\left[1-\Gamma_{0}(b)\right] \frac{i}{\omega} \frac{k_{z}^{2}}{k_{\perp}^{2}} \frac{e}{T_{\perp p}^{(0)}} \\
& \times\left[\frac{T_{\perp p}^{(0)}-T_{\| p}^{(0)}}{m_{p}}\left(1+\frac{k_{z}^{2}}{k_{\perp}^{2}}\right)(\Phi-\Psi)\right. \\
& \left.-\frac{\omega^{2}}{k_{z}^{2}}\left(\Phi+\frac{k_{z}^{2}}{k_{\perp}^{2}}(\Phi-\Psi)\right)\right] .
\end{aligned}
$$

On the other hand,

$$
\begin{aligned}
u_{z}= & \sqrt{\frac{2 T_{\|}^{(0)}}{m}}\left[\Gamma_{1}(b)-\Gamma_{0}(b)\right] \frac{T_{\perp}^{(0)}}{T_{\|}^{(0)}} \zeta R(\zeta) \frac{b_{z}}{B_{0}} \\
& -\sqrt{\frac{2 T_{\|}^{(0)}}{m}} \Gamma_{0}(b) \zeta R(\zeta) \frac{e \Psi}{T_{\|}^{(0)}}-\frac{k_{z}}{\omega} \frac{T_{\perp}^{(0)}-T_{\|}^{(0)}}{m} \\
& \times\left[1-\Gamma_{0}(b)\right] \frac{e}{T_{\perp}^{(0)}}\left(1+\frac{k_{z}^{2}}{k_{\perp}^{2}}\right)(\Phi-\Psi) .
\end{aligned}
$$

\section{Temperatures}

One has

$$
\begin{aligned}
\frac{T_{\perp p}^{(1)}}{T_{\perp p}^{(0)}}= & {\left[-2 b \Gamma_{1}(b)+2 b \Gamma_{0}(b)-\Gamma_{0}(b)\right]\left(\frac{T_{\perp p}^{(0)}}{T_{\| p}^{(0)}} R\left(\zeta_{p}\right)-1\right) \frac{b_{z}}{B_{0}} } \\
& -\left[b \Gamma_{1}(b)-b \Gamma_{0}(b)\right] R\left(\zeta_{p}\right) \frac{e \Psi}{T_{\| p}^{(0)}} \\
& +\left[b \Gamma_{1}(b)-b \Gamma_{0}(b)\right] \frac{e}{T_{\perp p}^{(0)}}\left(\Phi+\frac{k_{z}^{2}}{k_{\perp}^{2}}(\Phi-\Psi)\right)
\end{aligned}
$$

and

$$
\begin{aligned}
& \frac{T_{\| p}^{(1)}}{T_{\| p}^{(0)}}= {\left[1-R(\zeta)+2 \zeta^{2} R(\zeta)\right] \frac{T_{\perp} p^{(0)}}{T_{\| p}^{(0)}} } \\
& \times\left[\left[\Gamma_{1}(b)-\Gamma_{0}(b)\right] \frac{b_{z}}{B_{0}}-\Gamma_{0}(b) \frac{e \Psi}{T_{\perp p}^{(0)}}\right] .
\end{aligned}
$$

\section{Gyroviscous stress tensor}

One can show that 


$$
\begin{aligned}
\frac{\Pi_{x x}}{p_{\perp p}^{(0)}}= & -\cos 2 \psi\left[b \Gamma_{0}(b)-\Gamma_{1}(b)-b \Gamma_{1}(b)\right]\left[2\left(\frac{T_{\perp p}^{(0)}}{T_{\| p}^{(0)}} R\left(\zeta_{p}\right)-1\right) \frac{b_{z}}{B_{0}}+R\left(\zeta_{p}\right) \frac{e \Psi}{T_{\| p}^{(0)}}-\frac{e}{T_{\perp p}^{(0)}}\left(\Phi+\frac{k_{z}^{2}}{k_{\perp}^{2}}(\Phi-\Psi)\right)\right]-\cos 2 \psi \Gamma_{1}(b) \\
& \times\left(\frac{T_{\perp p}^{(0)}}{T_{\| p}^{(0)}} R\left(\zeta_{p}\right)-1\right) \frac{b_{z}}{B_{0}}+i \sin 2 \psi \frac{k_{z}}{\Omega} \frac{k_{z}}{\omega} \times \frac{e}{T_{\perp p}^{(0)}}\left[\left[\Gamma_{0}(b)-\Gamma_{1}(b)\right]-\frac{1}{b}\left[1-\Gamma_{0}(b)\right]\left[\frac{T_{\perp p}^{(0)}-T_{\| p}^{(0)}}{m_{p}}\left(1+\frac{k_{z}^{2}}{k_{\perp}^{2}}\right)(\Phi-\Psi)\right.\right. \\
& \left.-\frac{\omega^{2}}{k_{z}^{2}}\left(\Phi+\frac{k_{z}^{2}}{k_{\perp}^{2}}(\Phi-\Psi)\right)\right]-i \sin 2 \psi \frac{\omega}{\Omega}\left[\frac{1}{b}\left[\Gamma_{0}(b)-1-\Gamma_{1}(b)\right]+2\left[\Gamma_{0}(b)-\Gamma_{1}(b)\right]\right] \frac{b_{z}}{B_{0}},
\end{aligned}
$$

together with

$$
\begin{aligned}
\frac{\Pi_{x z}}{p_{\| p}^{(0)}}= & i \sin \psi \frac{T_{\perp}^{(0)}}{T_{\|}^{(0)}} \frac{k_{\perp}}{\Omega} \frac{\omega}{k_{z}}\left[\Gamma_{0}(b)-\Gamma_{1}(b)\right] R(\zeta)\left(2 \frac{T_{\perp}^{(0)}}{T_{\|}^{(0)}} \frac{b_{z}}{B_{0}}+\frac{e \Psi}{T_{\|}^{(0)}}\right)+\cos \psi \frac{k_{z}}{k_{\perp}}\left(\frac{T_{\perp}^{(0)}}{T_{\|}^{(0)}}-1\right)\left[\left[\Gamma_{0}(b)-\Gamma_{1}(b)-1\right] \frac{b_{z}}{B_{0}}\right. \\
& \left.-\left[1-\Gamma_{0}(b)\right] \frac{e \Psi}{T_{\perp}^{(0)}}\right]-\left[\cos \psi\left(\frac{T_{\perp}^{(0)}}{T_{\|}^{(0)}}-2\right) \frac{k_{z}}{k_{\perp}}\left[1-\Gamma_{0}(b)\right]+i \sin \psi \frac{T_{\perp}^{(0)}-T_{\|}^{(0)}}{m} \frac{T_{\perp}^{(0)}}{T_{\|}^{(0)}} \frac{k_{z} k_{\perp}}{\omega \Omega}\left[\Gamma_{0}(b)-\Gamma_{1}(b)-1\right]\right] \\
& \times\left(1+\frac{k_{z}^{2}}{k_{\perp}^{2}}\right) \frac{e}{T_{\perp}^{(0)}}(\Phi-\Psi)+4 i C_{1}^{3}(b) \sin \psi\left(\frac{T_{\perp}^{(0)}}{T_{\|}^{(0)}}-2\right) \frac{k_{z} \omega}{k_{\perp} \Omega} \frac{b_{z}}{B_{0}},
\end{aligned}
$$

where, since the last term is negligible in the spectral cone $k_{\|} / k_{\perp} \ll 1$, the coefficient $C_{1}^{3}(b)$, can be replaced by its limit value $1 / 4$ as $b \rightarrow 0$.

The elements $\Pi_{x y}$ and $\Pi_{y z}$ are deduced from $\Pi_{x x}$ and $\Pi_{x z}$, respectively, by replacing $\sin 2 \psi$ by $-\cos 2 \psi$ and $\cos 2 \psi$ by $\sin 2 \psi$.

\section{Heat fluxes}

One has

$$
\begin{aligned}
S_{x}^{\perp}= & p_{\perp}^{(0)} \frac{k_{\perp}}{\Omega} \frac{T_{\perp}^{(0)}}{m} i \sin \psi\left\{\left[2 \Gamma_{0}(b)-4 b \Gamma_{0}(b)+4 b \Gamma_{1}(b)\right]\left(\frac{T_{\perp}^{(0)}}{T_{\|}^{(0)}} R(\zeta)-1\right) \frac{b_{z}}{B_{0}}+\left[2 b \Gamma_{1}(b)-2 b \Gamma_{0}(b)+\Gamma_{1}(b)\right]\right. \\
& \left.\times\left[R(\zeta) \frac{e \Psi}{T_{\|}^{(0)}}-\frac{e}{T_{\perp}^{(0)}}\left(\Phi+\frac{k_{z}^{2}}{k_{\perp}^{2}}(\Phi-\Psi)\right)\right]\right\}+p_{\perp}^{(0)} \cos \psi\left\{-\frac{\omega}{k_{\perp}}\left[2 b \Gamma_{0}(b)-2 b \Gamma_{1}(b)-\Gamma_{1}(b)\right] \frac{b_{z}}{B_{0}}\right. \\
& +\frac{k_{z}^{2}}{k_{\perp} \omega}\left[\left(\frac{T_{\perp}^{(0)}-T_{\|}^{(0)}}{m}-\frac{\omega^{2}}{k_{z}^{2}}\right)\left[-1+\Gamma_{0}(b)+b \Gamma_{0}(b)-b \Gamma_{1}(b)\right] \frac{e}{T_{\perp}^{(0)}}\left(\Phi+\frac{k_{z}^{2}}{k_{\perp}^{2}}(\Phi-\Psi)\right)\right. \\
& \left.\left.-\frac{T_{\perp}^{(0)}-T_{\|}^{(0)}}{m}\left[-1+\Gamma_{0}(b)+b \Gamma_{0}(b)-b \Gamma_{1}(b)\right] \frac{e \Psi}{T_{\perp}^{(0)}}\right]\right\} .
\end{aligned}
$$

Similarly,

$$
\begin{aligned}
S_{x}^{\|}= & p_{\|}^{(0)}\left\{\frac{k_{\perp}}{\Omega} \frac{T_{\perp}^{(0)}}{m} i \sin \psi\left[\Gamma_{0}(b)-\Gamma_{1}(b)\right] \frac{T_{\perp}^{(0)}}{T_{\|}^{(0)}}[1-R(\zeta)\right. \\
& \left.+2 \zeta^{2} R(\zeta)\right]\left(\frac{e \Psi}{T_{\perp}^{(0)}}+2 \frac{b_{z}}{B_{0}}\right) \\
& -2 \frac{T_{\perp}^{(0)}-T_{\|}^{(0)}}{m} \frac{k_{z}^{2}}{k_{\perp} \Omega} i \sin \psi \frac{b_{z}}{B_{0}}+2 \frac{k_{z}^{2}}{\omega k_{\perp}} \cos \psi \frac{T_{\perp}^{(0)}-T_{\|}^{(0)}}{m} \\
& \left.\times\left[1-\Gamma_{0}(b)\right] \frac{e}{T_{\perp}^{(0)}}\left(1+\frac{k_{z}^{2}}{k_{\perp}^{2}}\right)(\Phi-\Psi)\right\} . \quad(\mathrm{B} 11)
\end{aligned}
$$

Furthermore, within the linear setting, $q_{\|}$and $q_{\perp}$ are approximated by

$$
\begin{aligned}
q_{\|} \approx S_{z}^{\|}= & -p_{\|}^{(0)} \sqrt{\frac{2 T_{\|}^{(0)}}{m}} \frac{T_{\perp}^{(0)}}{T_{\|}^{(0)}} \xi\left[1-3 R(\zeta)+2 \zeta^{2} R(\zeta)\right]\left[\left[\Gamma_{0}(b)\right.\right. \\
& \left.\left.-\Gamma_{1}(b)\right] \frac{b_{z}}{B_{0}}+\Gamma_{0}(b) \frac{e \Psi}{T_{\perp}^{(0)}}\right], \\
q_{\perp} \approx S_{z}^{\perp}= & p_{\perp}^{(0)}\left\{\frac{T_{\perp}^{(0)}}{T_{\|}^{(0)}} \frac{\omega}{k_{z}}\left[2 b \Gamma_{0}(b)-\Gamma_{0}(b)-2 b \Gamma_{1}(b)\right] R(\zeta) \frac{b_{z}}{B_{0}}\right. \\
& +\frac{\omega}{k_{z}} b\left[\Gamma_{0}(b)-\Gamma_{1}(b)\right] R(\zeta) \frac{e \Psi}{T_{\|}^{(0)}} \\
& -\frac{T_{\perp}^{(0)}-T_{\|}^{(0)}}{m} \frac{k_{z}}{\omega}\left[b \Gamma_{0}(b)-b \Gamma_{1}(b)\right] \frac{e}{T_{\perp}^{(0)}}\left(1+\frac{k_{z}^{2}}{k_{\perp}^{2}}\right) \\
& \times(\Phi-\Psi)\} .
\end{aligned}
$$




\section{Fourth-rank moments and cumulants}

The gyrotropic contribution to the fourth-order moment is characterized by

$$
\begin{aligned}
& r_{\perp \|}=p_{\perp}^{(0)} \frac{T_{\|}^{(0)}}{m}\left\{\left[2 b \Gamma_{0}(b)-2 \Gamma_{0}(b)-2 b \Gamma_{1}(b)+\Gamma_{1}(b)\right]\right. \\
& \times\left[\frac{T_{\perp}^{(0)}}{T_{\|}^{(0)}}-1+2 \frac{T_{\perp}^{(0)}}{T_{\|}^{(0)}} \zeta^{2} R(\zeta)\right] \frac{b_{z}}{B_{0}}+\frac{T_{\perp}^{(0)}}{T_{\|}^{(0)}}\left[b \Gamma_{0}(b)\right. \\
& \left.-\Gamma_{0}(b)-b \Gamma_{1}(b)\right]\left(1+2 \zeta^{2} R(\zeta)\right) \frac{e \Psi}{T_{\perp}^{(0)}}-\left[1-\Gamma_{0}(b)\right. \\
& \left.\left.+b \Gamma_{0}(b)-b \Gamma_{1}(b)\right] \frac{e}{T_{\perp}^{(0)}}\left(\Phi+\frac{k_{z}^{2}}{k_{\perp}^{2}}(\Phi-\Psi)\right)\right\}, \\
& r_{\|||}=p_{\|}^{(0)} \frac{T_{\|}^{(0)}}{m}\left\{[ \Gamma _ { 1 } ( b ) - \Gamma _ { 0 } ( b ) ] \left[3\left(\frac{T_{\perp}^{(0)}}{T_{\|}^{(0)}}-1\right)\right.\right. \\
& +2 \frac{T_{\perp}^{(0)}}{T_{\|}^{(0)}} \zeta^{2}\left[1+2 \zeta^{2} R(\zeta)\right] \frac{b_{z}}{B_{0}}-\Gamma_{0}(b) \frac{T_{\perp}^{(0)}}{T_{\|}^{(0)}} \\
& \times\left\{3+2 \zeta^{2}\left[1+2 \zeta^{2} R(\zeta)\right]\right\} \frac{e \Psi}{T_{\perp}^{(0)}}-3\left[1-\Gamma_{0}(b)\right] \frac{e}{T_{\perp}^{(0)}} \\
& \left.\times\left(\Phi+\frac{k_{z}^{2}}{k_{\perp}^{2}}(\Phi-\Psi)\right)\right\}, \\
& r_{\perp \perp}=p_{\perp}^{(0)} \frac{T_{\perp}^{(0)}}{m}\left\{\left[11 b \Gamma_{0}(b)-4 b^{2} \Gamma_{0}(b)-6 \Gamma_{0}(b)\right.\right. \\
& \left.-9 b \Gamma_{1}(b)+4 b^{2} \Gamma_{1}(b)+2 \Gamma_{1}(b)\right]\left(\frac{T_{\perp}^{(0)}}{T_{\|}^{(0)}} R(\zeta)-1\right) \frac{b_{z}}{B_{0}} \\
& -2\left[b^{2} \Gamma_{0}(b)-2 b \Gamma_{0}(b)+\Gamma_{0}(b)-b^{2} \Gamma_{1}(b)\right. \\
& \left.+\frac{3}{2} b \Gamma_{1}(b)\right] R(\zeta) \frac{e \Psi}{T_{\|}^{(0)}}+2\left[-1+b^{2} \Gamma_{0}(b)-2 b \Gamma_{0}(b)\right. \\
& \left.+\Gamma_{0}(b)-b \Gamma_{1}(b)+\frac{3}{2} b \Gamma_{1}(b)\right] \frac{e}{T_{\perp}^{(0)}} \\
& \left.\times\left(\Phi+\frac{k_{z}^{2}}{k_{\perp}^{2}}(\Phi-\Psi)\right)\right\} .
\end{aligned}
$$

After linearizing Eqs. (10)-(12), one gets for the cumulants

$$
\begin{aligned}
\tilde{r}_{\| \mid}= & \frac{p_{\|}^{(0)} T_{\perp}^{(0)}}{m}\left\{2 \zeta^{2}\left[1+2 \zeta^{2} R(\zeta)\right]+3[R(\zeta)-1]-12 \zeta^{2} R(\zeta)\right\} \\
& \times\left\{\left[\Gamma_{1}(b)-\Gamma_{0}(b)\right] \frac{b_{z}}{B_{0}}-\Gamma_{0}(b) \frac{e \Psi}{\left.T_{\perp}^{(0)}\right\},}\right. \\
\widetilde{r}_{\| \perp}= & \frac{p_{\perp}^{(0) 2}}{\rho^{(0)}}\left[1-R(\zeta)+2 \zeta^{2} R(\zeta)\right]\left\{\left[2 b \Gamma_{0}(b)-\Gamma_{0}(b)\right.\right. \\
& \left.\left.-2 b \Gamma_{1}(b)\right] \frac{b_{z}}{B_{0}}+b\left[\Gamma_{0}(b)-\Gamma_{1}(b)\right] \frac{e \Psi}{T_{\perp}^{(0)}}\right\},
\end{aligned}
$$

$$
\begin{aligned}
\widetilde{r}_{\perp \perp}= & \frac{p_{\perp}^{(0) 2}}{\rho^{(0)}}\left\{\left[4 b^{2} \Gamma_{1}(b)-4 b^{2} \Gamma_{0}(b)-b \Gamma_{1}(b)+3 b \Gamma_{0}(b)\right]\right. \\
& \times\left(\frac{T_{\perp}^{(0)}}{T_{\|}^{(0)}} R(\zeta)-1\right) \frac{b_{z}}{B_{0}}+\left[2 b^{2} \Gamma_{1}(b)+b \Gamma_{1}(b)\right. \\
& \left.-2 b^{2} \Gamma_{0}(b)\right] R(\zeta) \frac{e \Psi}{T_{\|}^{(0)}}+\left[2 b^{2} \Gamma_{0}(b)+2 b^{2} \Gamma_{1}(b)\right. \\
& \left.\left.-7 b \Gamma_{1}(b)\right] \frac{e}{T_{\perp}^{(0)}}\left(\Phi+\frac{k_{z}^{2}}{k_{\perp}^{2}}(\Phi-\Psi)\right)\right\} .
\end{aligned}
$$

${ }^{1}$ R. J. Leamon, C. W. Smith, N. F. Ness, W. H. Matthaeus, and H. K. Wong, J. Geophys. Res. 103, 4775 (1998).

${ }^{2}$ M. L. Golstein and A. Roberts, Phys. Plasmas 6, 4154 (1999).

${ }^{3}$ O. Alexandrova, A. Mangeney, M. Maksimovic, N. Cornilleau-Wehrlin, J. M. Bosqued, and M. André, J. Geophys. Res. 111, A12208, DOI:10.1029/ 2006JA011934 (2006).

${ }^{4}$ S. Galtier, J. Plasma Phys. 72, 721 (2001).

${ }^{5}$ S. Ghosh, E. Siregar, D. Q. Roberts, and M. L. Goldstein, J. Geophys. Res. 101, 2493 (1996).

${ }^{6}$ O. Alexandrova, V. Carbone, P. Veltri, and L. Sorriso-Valvo, "Solar wind turbulent spectra and role of Hall effect: Cluster observations," Planet. Space Sci. (in press).

${ }^{7}$ A. A. Schekochihin, S. C. Cowley, W. Dorland, G. W. Hammett, G. G. Howes, E. Quataert, and T. Tatsuno, "Kinetic and fluid turbulent cascades in magnetized weakly collisional astrophysical plasmas," Astrophys. J., Suppl. Ser. (submitted); e-print arXiv:astro-ph/0704.0044v1.

${ }^{8}$ S. R. Cranmer and A. A. van Ballegooijen, Astrophys. J. 591, 573 (2003).

${ }^{9}$ G. C. Howes, S. C. Cowley, W. Dorland, G. Hammett, E. Quataert, and A. A. Schekochihin, Astrophys. J. 651, 590 (2006).

${ }^{10}$ P. Goldreich and S. Sridhar, Astrophys. J. 485, 680 (1997).

${ }^{11}$ O. Stawicki, S. P. Gary, and H. Li, J. Geophys. Res. 106, 8273 (2001).

${ }^{12}$ G. Belmont, F. Sahraoui, and L. Rezeau, Adv. Space Res. 37, 1503 (2006).

${ }^{13}$ R. A. Treumann, Adv. Space Res. 37, 1482 (2006).

${ }^{14}$ J. Büchner, Astrophys. Space Sci. 264, 25 (1999).

${ }^{15}$ N. F. Loureiro, S. C. Cowley, W. D. Dorland, G. W. Hammett, and A. A. Schekochihin, "FLR effects in nonlinear tearing mode reconnection," in Proceedings of the 33rd EPS Conference on Plasma Physics, Rome, 19-23 June 2006, ECA 30I, p. 4.072.

${ }^{16}$ P. B. Snyder, G. W. Hammett, and W. Dorland, Phys. Plasmas 4, 3974 (1997).

${ }^{17}$ P. Goswami, T. Passot, and P. L. Sulem, Phys. Plasmas 12, 102109 (2005).

${ }^{18}$ A. Kritsuk, M. L. Norman, P. Padoan, and R. Wagner, "The statistics of supersonic isothermal turbulence," Astrophys. J. (submitted); e-print arXiv:astro-ph/0704.3851v1.

${ }^{19}$ J. J. Ramos, Phys. Plasmas 12, 052102 (2005).

${ }^{20}$ C. K. Hedrick and J. N. Leboeuf, Phys. Fluids B 4, 3915 (1992).

${ }^{21}$ T. Passot and P. L. Sulem, J. Geophys. Res. 111, A04203, DOI:10.1029/ 2005JA011425 (2006).

${ }^{22}$ T. Chust and G. Belmont, Phys. Plasmas 13, 012506 (2006).

${ }^{23} \mathrm{~K}$. Rönnmark, "Waves in homogeneous, anisotropic multicomponent plasmas (WHAMP)," Report No. 179, Kiruna Geophysical Institute, 1982; Plasma Phys. 25, 699 (1983).

${ }^{24}$ N. Yajima, Prog. Theor. Phys. 36, 1 (1966).

${ }^{25}$ D. Borgogno, T. Passot, and P. L. Sulem, Nonlinear Processes Geophys. 14, 373 (2007).

${ }^{26}$ E. A. Kuznetsov, T. Passot, and P. L. Sulem, Phys. Rev. Lett. 98, 235003 (2007).

${ }^{27}$ Y. Voitenko, J. Plasma Phys. 60, 497 (1998); 60, 515 (1998).

${ }^{28}$ Note that in some cases, particularly for large wavenumbers, the WHAMP program notified of possible inaccuracies in the results. We have nevertheless retained these values as they compared well with the model predictions. 